\title{
Central banks' preferences and banking sector vulnerability
}

\author{
G. Levieuge* Y. Lucotte $^{\dagger} \quad$ F. Pradines-Jobet ${ }^{\ddagger \S}$
}

October 23, 2017

\begin{abstract}
According to "Schwartz's conventional wisdom" and what has been called "divine coincidence", price stability should imply macroeconomic and financial stability. However, in light of the global financial crisis, with monetary policy focused on price stability, scholars have held that banking and financial risks were largely unaddressed. According to this alternative view, the belief in divine coincidence turns out to be benign neglect. The objective of this paper is to test Schwartz's hypothesis against the benign neglect hypothesis. The priority assigned to the inflation goal is proxied by the central banks' conservatism (CBC) index proposed by Levieuge and Lucotte (2014), here extended to a large sample of 73 countries from 1980 to 2012 . Banking sector vulnerability is measured by six alternative indicators that are frequently employed in the literature on early warning systems. Our results indicate that differences in monetary policy preferences robustly explain cross-country differences in banking vulnerability and validate the benign neglect hypothesis, in that a higher level of $\mathrm{CBC}$ implies a more vulnerable banking sector.
\end{abstract}

JEL Codes: E3; E44; E52; E58.

Keywords: Central Banks' Preferences, Inflation Aversion, Banking Sector Vulnerability, Monetary Policy.

*Banque de France DGEI-DEMFI-RECFIN (041-1391); 31, rue Croix des Petits Champs, 75049 Paris Cedex 01 France \& Univ. Orléans, CNRS, LEO, UMR 7322, F-45067, Orléans, France. Corresponding author. Email: gregory.levieuge@banque-france.fr

${ }^{\dagger}$ Univ. Orléans, CNRS, LEO, UMR 7322, F-45067, Orléans, France \& PSB Paris School of Business. E-mail: ylucotte@gmail.com

${ }^{\ddagger}$ Univ. Orléans, CNRS, LEO, UMR 7322, F-45067, Orléans, France. E-mail: florian.pradinesjobet@univ-orleans.fr

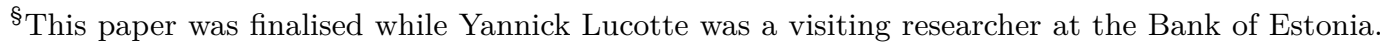
He would like to thank the Bank of Estonia for its hospitality and financial support. We also thank E. Farvaque, P.-G. Méon, R. Rovelli, J. Paez-Farrell, T. Rõõm, D. Kulikov, R. Bellando, and M. Širáňová for their comments and suggestions. We are indebted to the managing editor Iftekhar Hasan and the two anonymous referees for detailed comments and suggestions that led to considerable improvements in the paper. The views expressed in the article are those of the authors and do not necessarily reflect those of the Banque de France, the Bank of Estonia, or the Eurosystem. Any remaining errors are ours. 


\section{Introduction}

Since the public authorities in industrialized countries entrusted newly independent central banks with disinflation policies in the 1980s, price stability has become the main objective of monetary policy. The advent of the inflation targeting framework and the considerable support it has received among central bankers and academics can be viewed as the culmination of this orientation (King, 1997).

This top priority assigned to the control of inflation stems from the adherence of numerous economists and central bankers to Schwartz's "conventional wisdom" (Schwartz, 1995), according to which price stability implies macroeconomic and financial stability. It was widely accepted as a "divine coincidence" that having a monetary policy focused primarily on price stability would ensure output stability and maximum welfare, provided that distortions are composed solely of price rigidities (Woodford, 2003). The idea that price stability is a sufficient condition for guaranteeing financial stability was a leitmotiv in the 2000s. The conclusion of Bernanke and Gertler (2000, p.46) is representative of this perspective: "Given a strong commitment to stabilizing expected inflation, it is neither necessary nor desirable for monetary policy to respond to changes in asset prices, except to the extent that they help to forecast inflationary or deflationary pressures". The second part of this quote refers to the "Jackson Hole Consensus", which says that central banks should respond to financial developments only if they threaten price stability. In practice, this led most central banks to adopt a strategy of "cleaning up (the bust) afterwards", rather than a strategy of "leaning against the wind".

Certainly, a high level of inflation is not conducive to macroeconomic and financial stability. By showing that high-inflation countries are more subject to financial crises, some empirical studies such as Bordo et al. (2001) Bordo and Wheelock (1998) and Demirgüç-Kunt and Detragiache (1998) are in some ways in accordance with the Schwartz's conventional wisdom.

However, many recent financial crises were not preceded by periods of price instability (White, 2006), and typically, the recent financial crisis occurred in the context of the Great Moderation. This has shed some doubt on Schwartz's hypothesis. Many upstanding authors and institutions now argue that with monetary policies focused primarily on inflation, financial stability was largely ignored ${ }^{1}$. In turn, financial instability has undermined macroeconomic stability, despite a low and stable inflation rate. In this alternative view, the belief in the divine coincidence has, in retrospect, been revealed to be benign neglect. The following quotation of Mishkin (2017, p.256) is representative of this reversal: "central banks' success in stabilising inflation and the decreased volatility of business cycle fluctuations, which became known as the Great Moderation, made policy-makers complacent about the risks from financial disruptions. The benign economic environment leading up to 2007, however, did surely not protect the economy from financial instability. Indeed, it may have promoted it. Although price and output stability are surely beneficial, the recent crisis indicates that a policy focused solely on these objectives may not be enough to produce good economic outcomes".

This alternative view benefits from theoretical support. In particular, it can be demonstrated that the "divine coincidence" does not hold when real rigidities are present (Blanchard and Galí, 2007), as well as in the presence of financial imperfections (Lambertini et al., 2013; Reis, 2013; Woodford, 2012). Christiano et al. (2010)

\footnotetext{
${ }^{1}$ See for instance Bayoumi et al. (2014), Borio (2014b), Bernanke (2013) and Whelan (2013).
} 
show that as inflation is stable during periods of stock market booms while credit increases sharply, a central bank that focuses excessively on inflation overlooks the financial imbalances that such a policy exacerbates. Overall, because of its impact on welfare - beyond its effects on inflation and output forecasts - financial stability deserves to be a goal in itself. The problem is that monetary policy and financial stability policy may sometimes be conflicting and both may have negative externalities on each other ${ }^{2}$. This suggests the existence of a trade-off between those two objectives in certain circumstances. Given the legal mandates of central banks, priority is often given to the inflation goal to the detriment of financial stability.

On empirical grounds, to the best of our knowledge, only Blot et al. (2015) have recently addressed the issue of the Schwartz's hypothesis frontally. Using various methods, they reject the hypothesis that price stability is positively correlated with financial stability. Nevertheless, it is a policymakers' decision, namely their relative preferences and objectives, and not the inflation rate per se, which defines whether they turn away from the financial stability objective. As inflation is potentially subject to shocks and exogenous trends, beyond the will of policymakers, it only constitutes a rough proxy of what fundamentally underlies the Schwartz's hypothesis and the benign neglect hypothesis (i.e. policymakers' decision).

Against this background, the objective of the present paper is to extend this very scarce literature by testing the Schwartz hypothesis against the benign neglect hypothesis: Does assigning a higher priority to inflation stabilization reduces or increases the vulnerability of the banking sector? To this end, we go further than the existing evidence by directly addressing the link between policymakers' preferences and financial stability, with different methodologies, with a genuine measure of the preferences of central banks, and over a period that includes the global financial crisis (GFC hereafter) years.

The preference of central banks for price stability is proxied by the CONS index of central banks' conservatism (CBC), suggested by Levieuge and Lucotte (2014) and based on the Taylor curve (Taylor, 1979). We consider six alternative measures for banking sector vulnerability that are widely used in the literature on early warning systems as determinants of financial crises $^{3}$ : credit volatility, the credit-to-GDP gap, the credit-to-deposit ratio, nonperforming loans, the Z-score, and the capital-to-asset ratio. In essence, these factors primarily concern the credit cycle and the structure of the banks' balance sheets. Our results, from a sample of 73 countries over the period 1980-2012, indicate that the degree of CBC robustly explains banking sector vulnerability, which is in line with the benign neglect hypothesis. On this respect, if the inflation targeting (IT) framework implies a narrower focus on the inflation stabilization objective, our results are in line with papers concluding that IT has some adverse financial and real effects (Petreski, 2014; Frappa and Mésonnier, 2010; Lin, 2010).

The remainder of this paper is organized as follows. Section 2 reviews the Schwartz's and the benign neglect hypotheses. Section 3 is dedicated to the way we measure central banks' preferences, using the CONS index of CBC, which we extend to a broader set than that initially proposed by Levieuge and Lucotte (2014). Data for the dependent and control variables are also detailed in Section 3. Section 4 describes the methodology we implement and presents the results. Robustness checks are performed

\footnotetext{
${ }^{2}$ See Laséen et al. (2017) and Ioannidou (2005).

${ }^{3}$ See, e.g. Schularick and Taylor (2012).
} 
in Section 5. Section 6 concludes and discusses the implications and extensions of our results.

\section{The Schwartz's and benign neglect hypotheses: A re- view}

According to the Scwhartz's conventional wisdom, by focusing on the objective of price stability, policymakers contribute not only to achieving high levels of economic activity and employment, but also foster financial stability. The main reason is that inflation creates uncertainty and disturbs the information contained in prices. Future real returns of asset prices and investment are thus incorrectly valued. As a consequence, asset accumulation and lending decisions are imperfect. Finally, the banking sector stability is threatened by increasing non-performing loans and default risks. Conversely, price stability promotes a sound and appropriate intertemporal allocation of resources, and thus sound lending operations. This view has found a more formal theoretical underpinning through the so-called "divine coincidence": in the absence of real imperfections, stabilizing inflation in standard new Keynesian models is found equivalent to stabilizing the welfare-relevant output gap (Blanchard and Galí, 2007; Woodford, 2003).

However, real imperfections matter in practice, implying a trade-off between inflation and output. Furthermore, it has been proven that financial imperfections may reduce welfare by themselves and not only through their impact on output and inflation (Lambertini et al., 2013; Reis, 2013; Woodford, 2012). To this view, as reducing the effects of financial distortions makes the economy operate more efficiently, financial stability should be an objective on its own. A single inflation goal is not enough. Similarly, many authors and institutions have expressed their doubts about the conventional view in the wake of the GFC.

In practice, institutional and legal arrangements governing monetary policy in every country assign an overriding priority to the inflation stabilization objective. According to the comprehensive survey led by Oosterloo and de Haan (2004) and the exhaustive report published by the BIS (2009), the objectives and powers of the financial stability function are not clearly and explicitly stated in legal texts. Even when legal statutes mention a financial stability objective, the understanding of what it entails is quite diffuse. For instance, central banks are supposed to act in favour of "promoting" or "contributing to" financial stability ${ }^{4}$. Such extra-statutory statements assign little commitment and responsibility (see details in BIS, 2009, tab.2 p.30).

Certainly, the objectives of central banks change over time (Toniolo and White, 2015) and policymakers have realized that they should pay more attention to financial instability since 2008. However, this aspiration is still informal and superficial. In the most recent literature, it is still presented as an "ongoing" debate, with few practical changes up until now (Khan, 2017; Lombardi and Siklos, 2016; Koetter et al., 2014) . Finally, from the survey of Smaga (2013), we can learn that central banks that have an objective of financial stability do not even have an official definition for "Financial sta-

\footnotetext{
${ }^{4}$ Preserving financial stability is often considered to be a concern for central banks, or even one of their main functions, but only because they are responsible for the functioning of the payment system.

${ }^{5}$ There are very few exceptions. For example, the Financial Services Act (2012) gives to the Bank of England a clear set of statutory objectives for the supervision of the financial system. Switzerland and India have also reformed the mandates of their central banks.
} 
bility". This contrasts with the clarity and accountability surrounding the quantitative objective of price stability.

Complementary - de facto - arguments explain why central bankers may neglect financial developments. First, monetary policy is not the most efficient tool to ensure financial stability, as it does not only affect the specific financial sector where distortions have to be corrected but also many macroeconomic variables. Moreover, its impact on asset prices is uncertain. More generally, knowledge on financial stability is largely incomplete in terms of definition, measures, and adequate policies. Thus, responding to financial developments may harm the credibility of the monetary authorities, with the fear of financial dominance. Even an explicit dual mandate makes the credibility of the central bank vulnerable to a new time-inconsistency problem (Ueda and Valencia, 2014) and may compromise the independence of the central bank (Cukierman, 2011). Furthermore, such uncertainty may lead policymakers to be conservative - as expressed by Brainard (1967) - namely to neglect the financial stability issue ${ }^{6}$ (Lombardi and Siklos, 2016) and to give priority to the inflation goal instead.

In such a context, four arguments explain how and why strong preferences for price stability can lead to benign neglect and adversely affect financial stability.

(i) Financial stability may be neglected because of desynchronization between consumer prices and the financial cycle. The business cycle and the financial cycle are not perfectly aligned (Borio, 2014a). Thus, while tighter monetary policy may be required to burst an asset price bubble, it may not necessarily be justified in terms of inflation, as it was the case in 2002-2007. Given the legal arrangements mentioned earlier, desynchronization leads central banks to give priority to the price stability objective and neglect financial imbalances.

(ii) Financial instability is exacerbated by the risk-taking channel of monetary policy if inflation is low. The vast literature on the risk-taking channel argues that monetary policy can be responsible for an increase in systemic risk, if conducted regardless of any objective other than the inflation goal in the context of the Great Moderation ${ }^{7}$. Indeed, prioritizing the inflation stabilization objective when the inflation rate is very low leads central banks to conduct loose monetary policies over a prolonged period. Such policies have been blamed for lowering risk perceptions and increasing risk tolerance.

(iii) Financial stability suffers from the consequences of a conflict of objectives. Side effects and conflicts between monetary policy and financial-stability policy can occur ${ }^{8}$. Ioannidou (2005) for example highlights the conflict between monetary policy, which usually requires high real interest rates in order to fight inflation, and regulatory or supervisory policy, which is concerned about the adverse effects of higher interest rate on the solvency of the banking sector. The risk-taking channel of monetary policy is another example of side effects. Similarly, macroprudential tools impact credit growth and external imbalances with consequences for aggregate demand and

\footnotetext{
${ }^{6}$ Here, "financial" and "banking" are considered as synonyms when discussing vulnerability, stability, and so forth.

${ }^{7}$ See, among others, Jiménez et al. (2014) and Borio and Zhu (2012).

${ }^{8}$ Discussions on the trade-off between these two objectives are provided for example by Laséen et al. (2017), Gadanecz et al. (2015) and Issing (2003).
} 
ultimately for inflation. Examining the policy architecture of 35 countries, Chortareas et al. (2016) find that central banks serving both monetary and banking supervision functions are less conservative than those with a single price stability mandate. In this vein, Hasan and Mester (2008), Di Noia and Di Giorgio (1999), Goodhart and Schoenmaker (1995) and Heller (1991) unanimously find that countries whose central banks do not have supervisory duties have overall lower inflation rates. Similarly, Ioannidou (2005) finds that the Federal Reserve becomes less strict in bank supervision when it tightens monetary policy. One explanation is that the Federal Reserve compensates banks for the extra pressure it puts on them. When monetary and prudential policies are conducted by two distinct agencies, the conflict of objectives raises the risk of "push-me, pull-you" behaviour between policymakers ${ }^{9}$. While the literature is far from being clear-cut on the optimal policy-mix to be implemented, it is at least obvious that the optimal equilibrium depends on policymakers' preferences. Through a contract theory model, Franck and Krausz (2008) demonstrate that under a sound banking system, conservative parties with low inflation objectives find it appropriate to separate banking supervision from the conduct of monetary policy. A way to interpret their conclusion is to admit that conflicts of objectives are less likely to occur under a sound banking system. In contrast, when there is banking instability, a single agent is needed to internalize the external effects of both banking supervision and monetary policy.

(iv) More focus on output stabilization would imply more focus on the objective of financial stability. Asset price changes and financial shocks have an impact on economic activity through well-known channels: wealth effects, Tobin's $Q$ channel, the financial accelerator mechanism, the bank capital channel and the exchange rate channel. Thus, if central banks were more concerned with output stabilization, they would focus more on the financial stability objective, following the "leaning against the wind" strategy.

While the previous points explain a benign neglect attitude, a few recent papers consider that there is no trade-off between monetary and financial stability, and support the Schwartz's hypothesis. Investigating the interactions between monetary and macroprudential instruments, De Paoli and Paustian (2017) find that increased conservatism improves welfare. On empirical grounds, Fazio et al. (2015) find that countries with inflation targeting (IT) frameworks have more stable banking systems. They interpret this result as a validation of the conventional wisdom: by reducing the degree of inflation uncertainty, IT countries are able to grow more. Then, in line with Lucas (2000), this should contribute to the development of credit markets and consequently to the improvement of financial stability.

This calls for a more general empirical assessment about the impact of the priority assigned to the inflation goal on the banking sector vulnerability. Focusing on policymakers' preferences is required to shed a light on this issue.

\footnotetext{
${ }^{9}$ See Smets (2014). For a discussion on the "single entity" vs "coordinated" approach, see Lombardi and Siklos (2016).
} 


\section{Data}

This section describes in detail characteristics of the variables we use in our empirical analysis and presents the theoretical justifications for them.

Measuring central banks' preferences. Attempts to measure CBC are very scarce in the literature. They are inconvenient to expand in time and space, often time-invariant and model-dependent. These caveats are circumvented by the recent CONS indicator proposed by Levieuge and Lucotte (2014), which we expand in this paper. This indicator is based on the Taylor curve (Taylor, 1979), which precisely represents the trade-off between price and output volatility. It consists in measuring the relative importance assigned to the objective of inflation stabilization through the empirical variances of inflation and output gap, as detailed in Appendix 1.

As Levieuge and Lucotte (2014) argue, the CONS indicator has at least two main advantages. It is time-varying and model-independent. It does not impose any assumption about the monetary policy rule or strategy that a central bank follows. So it can assess the relative preferences of a central bank whatever monetary regime in place. These features are particularly important for our study, as we consider countries that have heterogeneous monetary policy practices, and monetary policy strategies have changed substantially around the world in recent decades. For example, a growing number of industrialized and emerging economies have abandoned monetary targeting and have instead adopted an inflation targeting framework. As shown in Levieuge and Lucotte (2013), these changes affect the degree of CBC. Finally, while Levieuge and Lucotte (2014) focus solely on the OECD countries from 1980 to 1998, we extend their index to a broader set of 73 countries, on an annual basis from 1980 to 2012, using the empirical variances of inflation and output gap computed over five-year rolling windows. Note that the CONS index lies between 0 and 1 . The higher $C O N S$ is, the more conservative the central bank is considered to be in the sense of Rogoff (1985), and the lower it is, the less conservative the central bank. An immediate way to assess the relevance of this extension is to examine the correlation between $C O N S$ and the average inflation rate. Figure A2 in Appendix 1 indicates that except for in the 1980s, the correlation is clearly negative.

Note that a movement in the CONS index might not always reflect a conscious desire by the central bank to change its behaviour through changes in preferences. In particular, such a shift may partly result from a combination of supply and demand shocks. These shocks are supposed to be addressed over the five-year rolling windows that we consider to compute CONS. Indeed, the main task of the central bank is to respond to shocks so as to meet its objectives. Nevertheless, to be as rigorous as possible, supply and demand shocks will be taken into account as control variables (for details see infra). Moreover, we will use an alternative measure of CBC, labelled CONS_W, which is the CONS index adjusted for demand and supply shocks. Details are provided in Appendix 1. While supply and demand shocks were expected to be particularly important in some emerging countries in our sample, CONS and CONS_W are highly correlated, as we can see in Figure A3 in Appendix 1.

The average values of CONS and CONS_W by decades, for all the countries in our sample, are reported in Table A1 in Appendix 2. Overall, we observe that central banks became more conservative from the 1980s to the 2000s. This is particularly striking for the OECD countries, for at least two reasons. First, over this period, a 
significant number of them had joined the European Monetary Union (EMU) with the prospect of adopting the euro. This involved reforms in central bank legislation by the euro candidates and their rallying to the leadership of the reputedly conservative Bundesbank (Siklos, 2002). Second, more than one-third of the OECD countries have adopted an inflation targeting regime since the early 1990s. This has increased their inflation aversion, as shown by Levieuge and Lucotte (2013). In contrast, no clear trend emerges for non-OECD countries, in which preferences are heterogeneous.

Measures of banking sector vulnerability. As there is no universally accepted empirical measure of banking sector vulnerability, we employ six alternative variables commonly used in the literature.

First, a simple way of measuring the potential effect of benign neglect on financing conditions and financial instability more generally is to focus on credit volatility. In essence, the higher the credit volatility, the more unstable financing is for households and firms. This variable is calculated as a five-year moving variance on quarterly credit data, which come from the International Monetary Fund's International Financial Statistics (IFS) database.

Our second measure is the credit-to-GDP gap. This is one of the most widely accepted proxies for banking and financial imbalances among policymakers and academics. It is designed to measure the size of the credit cycle, as the deviations of credit from the "normal" range of historical experience - and then to capture excess credit growth. As argued by Minsky (1972) and Kindleberger (1978), credit booms tend to sow the seeds of crises. A number of empirical papers show that indicators of excess credit growth are efficient at providing a leading signal of banking distress (see, e.g. Giese et al., 2014; Schularick and Taylor, 2012; Borio and Lowe, 2004). A case in point is Dell'Ariccia et al. (2014), who find that one third of credit booms are followed by crises and three-fifths are followed by a period of economic underperformance in the six years following the end of the boom. This empirical evidence certainly explains why the Basel Committee on Banking Supervision (BCBS) recommends using the credit-toGDP gap as a benchmark for the activation and release of the countercyclical capital buffer. We compute the credit-to-GDP gap as the difference between the credit-toGDP ratio and its Hodrick-Prescott (HP) filter trend. Credit refers to domestic loans provided by financial corporations to the household and private non-financial corporate sector. Data come from the World Bank's Global Financial Development (GFD) database.

The next four variables that we consider as proxies for banking sector vulnerability concern the structure of banks' balance sheets. They are taken from the GFD database. The first is the credit-to-deposit ratio, which measures the banking sector's funding stability. This ratio increases if credit creation is higher than deposit growth and decreases in the opposite case. Thus a higher ratio indicates there is more wholesale funding in the capital structure and is a signal of excessive bank leverage. As shown by Stremmel and Zsámboki (2015), an increasing credit-to-deposit ratio positively contributes to the amplitude of the financial cycle. Several recent papers about the global financial crisis indicate that the credit-to-deposit ratio is a good predictor of financial distress. For example, Caprio et al. (2014) show that the probability of suffering from the crisis in 2008 was larger for countries where the credit-to-deposit ratio was at higher levels. Ratnovski and Huang (2009) find that a large share of wholesale funding was the most robust predictor of distress for financial institutions 
during the subprime crisis.

Next, we consider the ratio of nonperforming loans to total gross loans. This variable is used as a proxy for the quality of banks' assets and, more generally, as a proxy for banking system stability. A higher value of this ratio indicates a degradation of the quality of the assets held by the banks in a given country. According to Čihák and Schaeck (2010), the proportion of nonperforming loans is also a good predictor of systemic banking vulnerabilities.

Then we consider the Z-score, a measure that is widely used in the literature to capture the solvency of the banking system (see, e.g. Beck et al., 2010; Laeven and Levine, 2009; Demirgüç-Kunt et al., 2008). It is based on a comparison between banks' buffers in the form of their capitalization and returns and their risks in the volatility of returns. Formally, the Z-score is defined as $Z=(k+\mu) / \sigma$, where $k$ is equity capital as a percentage of assets, $\mu$ is return as a percentage of assets, and $\sigma$ is the standard deviation of return on assets as a proxy for return volatility. Because a bank becomes insolvent when the value of its assets drops below the value of its debt, the Z-score can be interpreted as the number of standard deviations that a bank's return must fall below its expected value to wipe out all the equity in the bank and render it insolvent. The Z-score is inversely related to the probability of a bank becoming insolvent. As our empirical analysis is conducted at the country level, the Z-score can then be interpreted as the banking system's distance to default.

Our last measure of banking sector vulnerability is the bank capital-to-asset ratio, which measures the banking system's capitalization. A higher ratio indicates a better capitalized banking system. As a bank with higher capital provides a cushion against insolvency and better resilience to adverse shocks, this ratio can be viewed as an inverse proxy for banking system vulnerability.

Note that the credit-to-deposit ratio, the capital-to-asset ratio and the share of nonperforming loans to total gross loans are variables that belong to the "financial soundness indicators" of the International Monetary Fund. Ultimately, using these six different indicators allows us to consider all aspects of banking sector vulnerabilities.

Control variables. We also need to control for factors other than CBC that may impact banking sector vulnerabilities. There is no consensus in the empirical literature on the determinants of financial and banking imbalances. Following the literature on early warning indicators (see, e.g. Frankel and Saravelos, 2012), we therefore consider a large range of structural, cyclical and regulatory control variables.

The first set of these variables is intended to control for the economic conditions and shocks that the banking sector faces. To this end, we identify demand and supply shocks by applying the decomposition scheme suggested by Blanchard and Quah (1989) and consider the variance of these shocks as control variables. Like with the inflation and output gap volatilities used to compute the CONS index, the variance of shocks is calculated over five-year rolling windows. As argued by Levieuge and Lucotte (2014), it is also important to control for demand and supply shocks because they can impact the output gap and inflation variabilities, and thus the value of the CONS index. Thus, by considering the variance of demand and supply shocks, we control for inflation and output gap volatilities not necessarily reflecting a conscious willingness by the central bank to prioritize inflation stabilization. We then take the heterogeneity of the country sample into account by considering real GDP per capita as an indicator of the level of development. This variable is taken from the World Bank's World Development 
Indicators (WDI) database.

The second set of control variables is intended to capture the degree of banking competition because this can affect the risk-taking behaviour of financial intermediaries and, in turn, banking sector vulnerability. We measure the level of banking competition using two proxies commonly employed in the banking literature. The first is the Lerner index, which measures the degree of market power of the banks and is thus an inverse proxy for bank competition. A low value (the minimum is 0 ) indicates a high degree of competition, while a high value (the maximum is 1 ) indicates a low competitive environment. The second proxy we consider is a measure of bank concentration. This corresponds to the assets of the three largest commercial banks as a share of total commercial banking assets. As with the Lerner index, bank concentration is an inverse proxy for competition because a concentrated market structure is expected to be associated with higher prices and profits, reflecting an uncompetitive context. These two variables are obtained from the GFD database. Despite the large number of studies devoted to the competition-stability nexus, the relationship between competition and bank risk-taking remains ambiguous. Under the "competition-fragility" view, bank competition is seen as detrimental to financial stability. Conversely, the "competition-stability" view rejects the competition-stability trade-off hypothesis and argues that market power increases bank portfolio risks.

Finally, we control for the regulation of the banking system. To this end, we consider the banking sector supervision index developed by Abiad et al. (2010). This index comprises four sub-components and takes values from 0 to 6 . A higher value indicates greater supervision and regulation of the banking system, then we expect that this variable is negatively related to the fragility of the banking sector. To have a complete picture of the degree of financial liberalization, we also consider a measure of financial openness using the Chinn-Ito index (Chinn and Ito, 2008). This index is a de jure measure of financial openness that assesses the extent of openness in capital account transactions. It is normalized between 0 and 1 , with the highest degree of financial openness corresponding to a value of 1 and the lowest to a value of 0 . The expected impact of this variable on the vulnerability of the banking sector is uncertain. On the one hand, according to Abiad et al. (2007), greater financial openness allows investors to diversify their portfolios: this implies a longer investment horizon and reduces the risk of sudden stops, which may in itself reduce banking vulnerability ${ }^{10}$. On the other hand, globally integrated financial systems are more exposed to international financial shocks and may experience more pronounced financial vulnerability (Giannone et al., 2011).

Figure 1 reports the mean value of our six measures of vulnerability for each quartile of the CBC indexes. We observe a positive correlation between the CBC indexes and the mean values of 1) credit volatility, 2) the credit-to-GDP gap, and 3) the credit-todeposit ratio, in accordance with the benign neglect hypothesis. Analogously, we see that higher degrees of conservatism are related to lower capital-to-asset ratios. The plots are less clear for the nonperforming loans ratio and the Z-score variable. We formally investigate this issue in the next section.

\footnotetext{
${ }^{10}$ See also Abiad et al. (2009) and Calvo et al. (2008) for empirical evidence.
} 


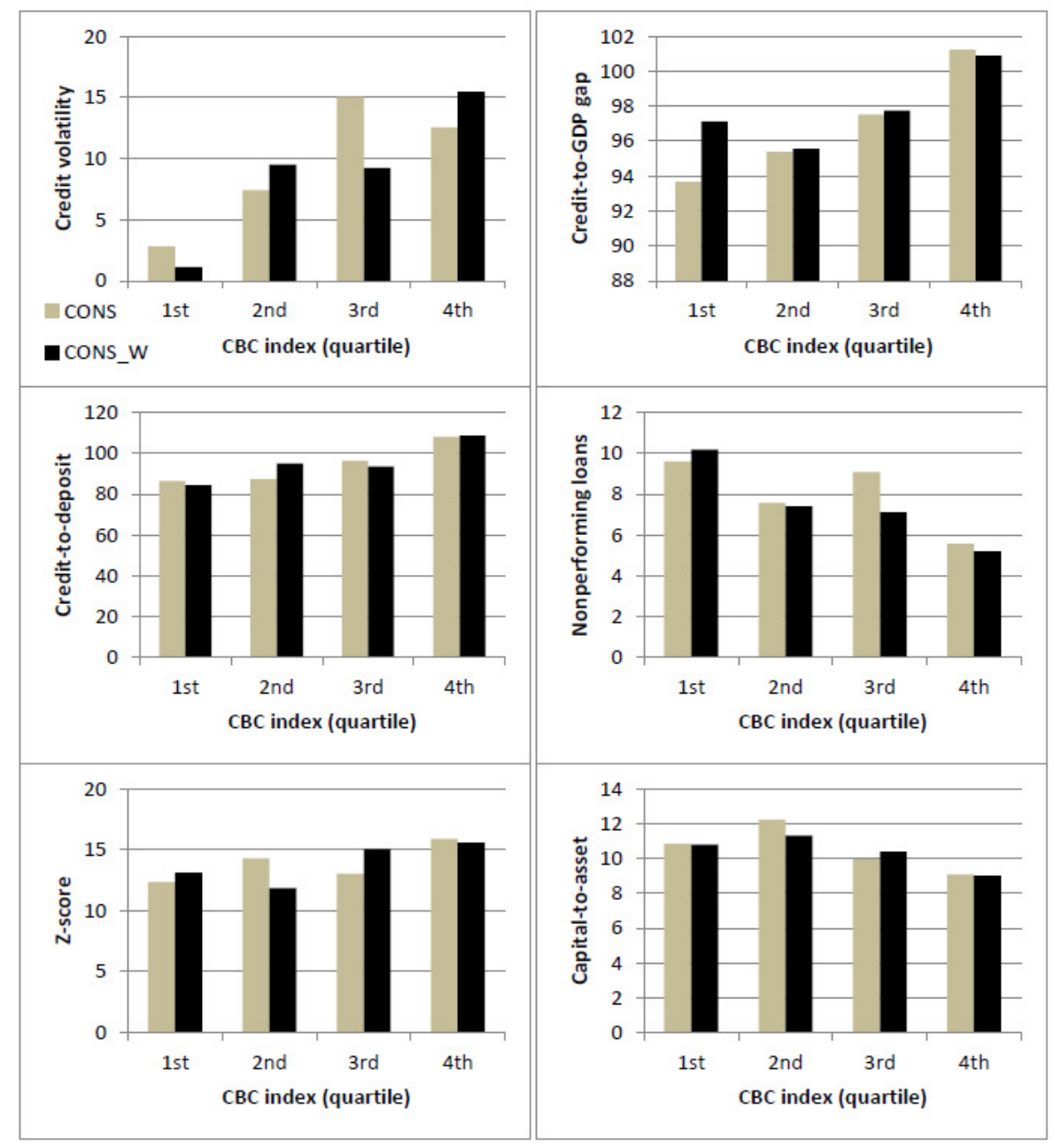

Figure 1: Central banks' preferences and banking sector vulnerability

\section{Methodology and results}

This section presents the methodology and the results of our empirical analysis. Driven by data availability, the sample covers 73 countries, from 1980 to $2012^{11}$. To test the impact of central banks' preferences on banking sector vulnerability, so testing benign neglect against Schwartz's hypothesis, we run the following estimation:

$$
Y_{i, t}=\alpha+\beta C B P_{i, t}+\gamma_{1} \sigma_{i, t}+\gamma_{2} X_{i, t-1}+\delta_{i}+\delta_{t}+\epsilon_{i, t}
$$

where $Y_{i t}$ alternatively represents one of our six measures of banking sector vulnerability for country $i$ at time $t$. $C B P_{i, t}$ is the indicator of central banks' preferences $(C O N S \text { or CONS_W })^{12}, \sigma_{i, t}$ is a vector containing the variances of supply and demand shocks, and $X_{i, t-1}$ is a vector that includes the other control variables, which

\footnotetext{
${ }^{11}$ See Appendix 2 for further details on the composition of our sample. Countries are excluded from the sample once they join a monetary union. This is the case for the members of the EMU, CEMAC, WAEMU and ECCU.

${ }^{12}$ As mentioned above, CONS and CONS_W are calculated using inflation and output gap volatilities computed over five-year rolling windows.
} 
are lagged to address potential endogeneity. Moreover, country fixed effects $\left(\delta_{i}\right)$ are included in equation (1) and are intended to eliminate unobserved time-invariant heterogeneity at the country level. We also introduce time fixed effects $\left(\delta_{t}\right)$ to absorb the impact of global shocks that may affect all the countries in the sample, such as the subprime crisis. $\epsilon_{i, t}$ is the error term.

Throughout the study, we will be particularly interested in the sign and significance of $\beta$. For $Y$, measuring banking sector vulnerabilities, a positive $\beta$ would validate the benign neglect hypothesis, while a negative one would support Schwartz's hypothesis. As the Z-score and capital-to-asset ratio are inverse proxies for banking vulnerabilities, the signs related to the alternative hypotheses are reversed.

Table 1 presents the results with credit volatility and the credit-to-GDP gap as endogenous variables. Table 2 reports results obtained with the credit-to-deposit ratio and the nonperforming loans to total gross loans ratio. Finally, Table 3 gives the results obtained with the Z-score and the capital-to-assets ratio as proxies for banking sector vulnerability. In each table, specification (1) includes CONS, the variances of macroeconomic shocks and real GDP per capita as explanatory variables. Specifications (2) and (3) then successively include variables intended to control for banking competition or concentration in (2), and for the financial environment in (3). Banking competition and banking concentration are included simultaneously because many studies find no evidence that bank competitiveness measures are related to banking system concentration (see, e.g. Claessens and Laeven, 2004). 
Table 1: Central banks' preferences and banking sector vulnerability: Results obtained with the credit volatility and the credit-to-GDP gap

\begin{tabular}{|c|c|c|c|c|c|c|}
\hline \multirow[t]{2}{*}{ Dependent variable } & \multicolumn{3}{|c|}{ Credit volatility } & \multicolumn{3}{|c|}{ Credit-to-GDP gap } \\
\hline & $(1)$ & $(2)$ & $(3)$ & $(1)$ & $(2)$ & $(3)$ \\
\hline CONS & $\begin{array}{c}21.876^{* *} \\
(11.009)\end{array}$ & $\begin{array}{c}62.121^{* * *} \\
(20.210)\end{array}$ & $\begin{array}{c}45.351^{* * *} \\
(13.176)\end{array}$ & $\begin{array}{c}15.300^{* * *} \\
(2.715)\end{array}$ & $\begin{array}{c}17.241^{* * *} \\
(3.534)\end{array}$ & $\begin{array}{c}14.845^{* * *} \\
(5.590)\end{array}$ \\
\hline Variance of supply shocks & $\begin{array}{l}-2.497 \\
(4.396)\end{array}$ & $\begin{array}{l}-1.610 \\
(8.524)\end{array}$ & $\begin{array}{l}-1.404 \\
(5.138)\end{array}$ & $\begin{array}{c}0.845 \\
(1.083)\end{array}$ & $\begin{array}{l}-1.983 \\
(1.478)\end{array}$ & $\begin{array}{l}-1.829 \\
(2.181)\end{array}$ \\
\hline Variance of demand shocks & $\begin{array}{c}6.219 \\
(4.200)\end{array}$ & $\begin{array}{c}5.836 \\
(7.436)\end{array}$ & $\begin{array}{c}6.038 \\
(5.355)\end{array}$ & $\begin{array}{c}-2.995^{* * *} \\
(1.033)\end{array}$ & $\begin{array}{c}-1.601 \\
(1.290)\end{array}$ & $\begin{array}{c}-4.482^{* *} \\
(2.267)\end{array}$ \\
\hline GDP per capita & $\begin{array}{l}-0.051 \\
(0.097)\end{array}$ & $\begin{array}{l}-0.220 \\
(0.236)\end{array}$ & $\begin{array}{l}-0.136 \\
(0.184)\end{array}$ & $\begin{array}{c}0.018 \\
(0.025)\end{array}$ & $\begin{array}{c}0.224^{* * *} \\
(0.044)\end{array}$ & $\begin{array}{c}0.489^{* * *} \\
(0.078)\end{array}$ \\
\hline Lerner index & & $\begin{array}{c}-71.759^{* *} \\
(32.971)\end{array}$ & $\begin{array}{c}-47.322^{* *} \\
(20.100)\end{array}$ & & $\begin{array}{c}16.776^{* * *} \\
(5.980)\end{array}$ & $\begin{array}{c}8.992 \\
(8.535)\end{array}$ \\
\hline Bank concentration & & $\begin{array}{c}0.001 \\
(0.269)\end{array}$ & $\begin{array}{l}-0.097 \\
(0.168)\end{array}$ & & $\begin{array}{c}0.025 \\
(0.050)\end{array}$ & $\begin{array}{l}-0.016 \\
(0.071)\end{array}$ \\
\hline Financial openness & & & $\begin{array}{c}-7.794 \\
(19.617)\end{array}$ & & & $\begin{array}{c}15.387^{*} \\
(8.319)\end{array}$ \\
\hline Banking supervision & & & $\begin{array}{l}-6.483 \\
(7.647)\end{array}$ & & & $\begin{array}{c}0.798 \\
(3.247)\end{array}$ \\
\hline Constant & $\begin{array}{c}3.171 \\
(50.881)\end{array}$ & $\begin{array}{c}3.588 \\
(37.540)\end{array}$ & $\begin{array}{c}21.713 \\
(39.302)\end{array}$ & $\begin{array}{c}20.089 \\
(13.857)\end{array}$ & $\begin{array}{c}-34.071^{* * *} \\
(6.782)\end{array}$ & $\begin{array}{c}-82.981^{* * *} \\
(16.826)\end{array}$ \\
\hline Observations & 874 & 520 & 339 & 998 & 628 & 343 \\
\hline R-squared & 0.047 & 0.071 & 0.089 & 0.144 & 0.230 & 0.275 \\
\hline Number of countries & 73 & 65 & 53 & 73 & 66 & 53 \\
\hline CONS_W & $\begin{array}{c}27.396^{* *} \\
(10.764)\end{array}$ & $\begin{array}{c}65.735^{* * *} \\
(20.449)\end{array}$ & $\begin{array}{c}45.038^{* * *} \\
(13.012)\end{array}$ & $\begin{array}{c}12.634^{* * *} \\
(2.682)\end{array}$ & $\begin{array}{c}15.400^{* * *} \\
(3.589)\end{array}$ & $\begin{array}{c}15.426^{* * *} \\
(5.507)\end{array}$ \\
\hline Variance of supply shocks & $\begin{array}{c}1.098 \\
(4.672)\end{array}$ & $\begin{array}{c}7.248 \\
(8.974)\end{array}$ & $\begin{array}{c}4.803 \\
(5.506)\end{array}$ & $\begin{array}{c}2.444^{* *} \\
(1.153)\end{array}$ & $\begin{array}{c}0.127 \\
(1.553)\end{array}$ & $\begin{array}{c}0.313 \\
(2.336)\end{array}$ \\
\hline Variance of demand shocks & $\begin{array}{c}2.797 \\
(4.350)\end{array}$ & $\begin{array}{l}-3.392 \\
(7.807)\end{array}$ & $\begin{array}{l}-1.101 \\
(5.422)\end{array}$ & $\begin{array}{c}-4.763^{* * *} \\
(1.064)\end{array}$ & $\begin{array}{c}-3.878^{* * *} \\
(1.333)\end{array}$ & $\begin{array}{c}-6.886^{* * *} \\
(2.294)\end{array}$ \\
\hline GDP per capita & $\begin{array}{c}-0.056 \\
(0.096)\end{array}$ & $\begin{array}{l}-0.205 \\
(0.235)\end{array}$ & $\begin{array}{l}-0.117 \\
(0.183)\end{array}$ & $\begin{array}{c}0.026 \\
(0.025)\end{array}$ & $\begin{array}{c}0.235^{* * *} \\
(0.044)\end{array}$ & $\begin{array}{c}0.493^{* * *} \\
(0.078)\end{array}$ \\
\hline Lerner index & & $\begin{array}{l}-64.512^{*} \\
(32.924)\end{array}$ & $\begin{array}{c}-43.986^{* *} \\
(20.013)\end{array}$ & & $\begin{array}{c}18.534^{* * *} \\
(6.045)\end{array}$ & $\begin{array}{l}10.009 \\
(8.488)\end{array}$ \\
\hline Bank concentration & & $\begin{array}{c}0.020 \\
(0.269)\end{array}$ & $\begin{array}{l}-0.112 \\
(0.168)\end{array}$ & & $\begin{array}{c}0.027 \\
(0.051)\end{array}$ & $\begin{array}{l}-0.019 \\
(0.071)\end{array}$ \\
\hline Financial openness & & & $\begin{array}{c}-7.844 \\
(19.612)\end{array}$ & & & $\begin{array}{l}15.369^{*} \\
(8.307)\end{array}$ \\
\hline Banking supervision & & & $\begin{array}{l}-5.719 \\
(7.632)\end{array}$ & & & $\begin{array}{c}1.032 \\
(3.237)\end{array}$ \\
\hline Constant & $\begin{array}{c}2.200 \\
(50.694)\end{array}$ & $\begin{array}{c}-0.826 \\
(37.796)\end{array}$ & $\begin{array}{c}20.474 \\
(39.342)\end{array}$ & $\begin{array}{c}22.115 \\
(13.914)\end{array}$ & $\begin{array}{c}-33.681^{* * *} \\
(6.918)\end{array}$ & $\begin{array}{c}-83.725^{* * *} \\
(16.824)\end{array}$ \\
\hline Observations & 874 & 520 & 339 & 998 & 628 & 343 \\
\hline R-squared & 0.050 & 0.073 & 0.089 & 0.135 & 0.223 & 0.277 \\
\hline Number of countries & 73 & 65 & 53 & 73 & 66 & 53 \\
\hline
\end{tabular}

Note: Standard errors are reported in parentheses. *; **, and *** indicate statistical significance at the $10 \%, 5 \%$ and $1 \%$ level, respectively. 
Table 2: Central banks' preferences and banking sector vulnerability: Results obtained with the credit-to-deposit ratio and the nonperforming loans ratio

\begin{tabular}{|c|c|c|c|c|c|c|}
\hline \multirow[t]{2}{*}{ Dependent variable } & \multicolumn{3}{|c|}{ Credit-to-deposit ratio } & \multicolumn{3}{|c|}{ Nonperforming loans ratio } \\
\hline & (1) & $(2)$ & (3) & $(1)$ & (2) & $(3)$ \\
\hline CONS & $\begin{array}{c}18.919^{* * *} \\
(5.451)\end{array}$ & $\begin{array}{c}31.487^{* * *} \\
(6.617)\end{array}$ & $\begin{array}{c}23.641^{* *} \\
(10.693)\end{array}$ & $\begin{array}{c}6.539^{* * *} \\
(1.378)\end{array}$ & $\begin{array}{c}6.692^{* * *} \\
(1.414)\end{array}$ & $\begin{array}{c}4.875^{* * *} \\
(1.866)\end{array}$ \\
\hline Variance of supply shocks & $\begin{array}{c}-10.270^{* * *} \\
(2.179)\end{array}$ & $\begin{array}{l}-3.088 \\
(2.692)\end{array}$ & $\begin{array}{l}-3.732 \\
(4.102)\end{array}$ & $\begin{array}{c}0.705 \\
(0.499)\end{array}$ & $\begin{array}{c}0.592 \\
(0.558)\end{array}$ & $\begin{array}{c}0.309 \\
(0.689)\end{array}$ \\
\hline Variance of demand shocks & $\begin{array}{c}-3.470^{*} \\
(2.097)\end{array}$ & $\begin{array}{c}-4.756^{*} \\
(2.443)\end{array}$ & $\begin{array}{c}-13.156^{* * *} \\
(4.248)\end{array}$ & $\begin{array}{c}2.354^{* * *} \\
(0.479)\end{array}$ & $\begin{array}{c}2.242^{* * *} \\
(0.493)\end{array}$ & $\begin{array}{c}2.237^{* * * *} \\
(0.716)\end{array}$ \\
\hline GDP per capita & $\begin{array}{c}0.317^{* * *} \\
(0.050)\end{array}$ & $\begin{array}{c}0.462^{* * *} \\
(0.085)\end{array}$ & $\begin{array}{c}0.993^{* * *} \\
(0.163)\end{array}$ & $\begin{array}{c}0.082^{* * *} \\
(0.017)\end{array}$ & $\begin{array}{c}0.074^{* * *} \\
(0.017)\end{array}$ & $\begin{array}{c}0.035 \\
(0.026)\end{array}$ \\
\hline Lerner index & & $\begin{array}{c}8.420 \\
(10.943)\end{array}$ & $\begin{array}{c}-20.553 \\
(16.185)\end{array}$ & & $\begin{array}{c}-10.156^{* * *} \\
(2.266)\end{array}$ & $\begin{array}{c}-7.782^{* * *} \\
(2.722)\end{array}$ \\
\hline Bank concentration & & $\begin{array}{c}-0.197^{* *} \\
(0.093)\end{array}$ & $\begin{array}{c}-0.309^{* *} \\
(0.144)\end{array}$ & & $\begin{array}{l}-0.022 \\
(0.019)\end{array}$ & $\begin{array}{c}0.025 \\
(0.023)\end{array}$ \\
\hline Financial openness & & & $\begin{array}{l}-21.987 \\
(15.634)\end{array}$ & & & $\begin{array}{l}-1.623 \\
(2.753)\end{array}$ \\
\hline Banking supervision & & & $\begin{array}{l}-2.668 \\
(6.188)\end{array}$ & & & $\begin{array}{l}-0.655 \\
(1.046)\end{array}$ \\
\hline Constant & $\begin{array}{c}23.120 \\
(26.760)\end{array}$ & $\begin{array}{c}51.461^{* * *} \\
(12.834) \\
\end{array}$ & $\begin{array}{c}11.704 \\
(31.951)\end{array}$ & $\begin{array}{c}-11.083^{* * *} \\
(2.634) \\
\end{array}$ & $\begin{array}{c}-7.613^{* *} \\
(2.976)\end{array}$ & $\begin{array}{l}-3.424 \\
(5.399) \\
\end{array}$ \\
\hline Observations & 940 & 581 & 325 & 607 & 574 & 316 \\
\hline R-squared & 0.150 & 0.192 & 0.235 & 0.303 & 0.331 & 0.396 \\
\hline Number of countries & 72 & 65 & 52 & 65 & 63 & 50 \\
\hline CONS_W & $\begin{array}{c}13.406^{* *} \\
(5.359)\end{array}$ & $\begin{array}{c}25.630^{* * *} \\
(6.694)\end{array}$ & $\begin{array}{c}25.961^{* *} \\
(10.501)\end{array}$ & $\begin{array}{c}6.328^{* * * *} \\
(1.409)\end{array}$ & $\begin{array}{c}5.961^{* * *} \\
(1.460)\end{array}$ & $\begin{array}{c}4.951^{* * *} \\
(1.882)\end{array}$ \\
\hline Variance of supply shocks & $\begin{array}{c}-8.614^{* * *} \\
(2.302)\end{array}$ & $\begin{array}{c}0.426 \\
(2.818)\end{array}$ & $\begin{array}{c}-0.172 \\
(4.360)\end{array}$ & $\begin{array}{c}1.575^{* * *} \\
(0.525)\end{array}$ & $\begin{array}{c}1.404^{* *} \\
(0.584)\end{array}$ & $\begin{array}{c}0.972 \\
(0.732)\end{array}$ \\
\hline Variance of demand shocks & $\begin{array}{c}-5.529^{* *} \\
(2.159)\end{array}$ & $\begin{array}{c}-8.945^{* * *} \\
(2.534)\end{array}$ & $\begin{array}{c}-17.119^{* * *} \\
(4.310)\end{array}$ & $\begin{array}{c}1.412^{* * *} \\
(0.486)\end{array}$ & $\begin{array}{c}1.364^{* * *} \\
(0.508)\end{array}$ & $\begin{array}{l}1.435^{*} \\
(0.737)\end{array}$ \\
\hline GDP per capita & $\begin{array}{c}0.327^{* * *} \\
(0.050)\end{array}$ & $\begin{array}{c}0.474^{* * *} \\
(0.086)\end{array}$ & $\begin{array}{c}0.996^{* * *} \\
(0.162)\end{array}$ & $\begin{array}{c}0.084^{* * *} \\
(0.017)\end{array}$ & $\begin{array}{c}0.077^{* * *} \\
(0.017)\end{array}$ & $\begin{array}{c}0.038 \\
(0.026)\end{array}$ \\
\hline Lerner index & & $\begin{array}{c}11.092 \\
(11.111)\end{array}$ & $\begin{array}{l}-19.245 \\
(16.100)\end{array}$ & & $\begin{array}{c}-9.510^{* * *} \\
(2.290)\end{array}$ & $\begin{array}{c}-7.493^{\text {*** }} \\
(2.706)\end{array}$ \\
\hline Bank concentration & & $\begin{array}{c}-0.198^{* *} \\
(0.094)\end{array}$ & $\begin{array}{c}-0.316^{* *} \\
(0.143)\end{array}$ & & $\begin{array}{c}-0.020 \\
(0.019)\end{array}$ & $\begin{array}{c}0.025 \\
(0.023)\end{array}$ \\
\hline Financial openness & & & $\begin{array}{l}-21.980 \\
(15.598)\end{array}$ & & & $\begin{array}{l}-1.749 \\
(2.752)\end{array}$ \\
\hline Banking supervision & & & $\begin{array}{l}-2.243 \\
(6.165)\end{array}$ & & & $\begin{array}{l}-0.577 \\
(1.044)\end{array}$ \\
\hline Constant & $\begin{array}{c}27.201 \\
(26.802)\end{array}$ & $\begin{array}{c}55.650^{* * *} \\
(13.049)\end{array}$ & $\begin{array}{c}10.082 \\
(31.869)\end{array}$ & $\begin{array}{c}-10.884^{* * *} \\
(2.642)\end{array}$ & $\begin{array}{c}-7.350^{* *} \\
(3.026)\end{array}$ & $\begin{array}{l}-3.621 \\
(5.410)\end{array}$ \\
\hline Observations & 940 & 581 & 325 & 607 & 574 & 316 \\
\hline R-squared & 0.144 & 0.179 & 0.239 & 0.300 & 0.323 & 0.396 \\
\hline Number of countries & 72 & 65 & 52 & 65 & 63 & 50 \\
\hline
\end{tabular}

Note: Standard errors are reported in parentheses. ${ }^{*} ; * *$, and $* * *$ indicate statistical significance at the $10 \%, 5 \%$ and $1 \%$ level, respectively. 
Table 3: Central banks' preferences and banking sector vulnerability: Results obtained with the Z-score and the capital-to-asset ratio

\begin{tabular}{|c|c|c|c|c|c|c|}
\hline \multirow[t]{2}{*}{ Dependent variable } & \multicolumn{3}{|c|}{ Z-score } & \multicolumn{3}{|c|}{ Capital-to-asset ratio } \\
\hline & $(1)$ & $(2)$ & $(3)$ & $(1)$ & $(2)$ & $(3)$ \\
\hline CONS & $\begin{array}{c}-2.273^{* *} \\
(1.105)\end{array}$ & $\begin{array}{c}-2.062^{*} \\
(1.200)\end{array}$ & $\begin{array}{c}-4.421^{*} \\
(2.298)\end{array}$ & $\begin{array}{c}-2.936^{* * *} \\
(0.598)\end{array}$ & $\begin{array}{c}-1.994^{* * *} \\
(0.580)\end{array}$ & $\begin{array}{l}-1.158 \\
(1.051)\end{array}$ \\
\hline Variance of supply shocks & $\begin{array}{c}0.691 \\
(0.438)\end{array}$ & $\begin{array}{c}0.374 \\
(0.495)\end{array}$ & $\begin{array}{l}-0.210 \\
(0.897)\end{array}$ & $\begin{array}{l}0.409^{*} \\
(0.211)\end{array}$ & $\begin{array}{c}0.199 \\
(0.227)\end{array}$ & $\begin{array}{l}-0.574 \\
(0.396)\end{array}$ \\
\hline Variance of demand shocks & $\begin{array}{c}-1.604^{* * *} \\
(0.408)\end{array}$ & $\begin{array}{c}-1.630^{* * *} \\
(0.433)\end{array}$ & $\begin{array}{c}-2.754^{* * *} \\
(0.932)\end{array}$ & $\begin{array}{c}-0.588^{* * *} \\
(0.204)\end{array}$ & $\begin{array}{c}-0.715^{* * *} \\
(0.194)\end{array}$ & $\begin{array}{c}-0.752^{*} \\
(0.386)\end{array}$ \\
\hline GDP per capita & $\begin{array}{l}-0.015 \\
(0.013)\end{array}$ & $\begin{array}{l}-0.023 \\
(0.015)\end{array}$ & $\begin{array}{l}-0.013 \\
(0.032)\end{array}$ & $\begin{array}{l}-0.013 \\
(0.008)\end{array}$ & $\begin{array}{c}-0.016^{* *} \\
(0.008)\end{array}$ & $\begin{array}{l}-0.026 \\
(0.018)\end{array}$ \\
\hline Lerner index & & $\begin{array}{c}2.911 \\
(2.036)\end{array}$ & $\begin{array}{l}-1.724 \\
(3.509)\end{array}$ & & $\begin{array}{c}4.067^{* * *} \\
(0.948)\end{array}$ & $\begin{array}{l}2.762^{*} \\
(1.513)\end{array}$ \\
\hline Bank concentration & & $\begin{array}{c}0.017 \\
(0.017)\end{array}$ & $\begin{array}{l}-0.010 \\
(0.029)\end{array}$ & & $\begin{array}{c}0.025^{* * *} \\
(0.008)\end{array}$ & $\begin{array}{c}0.043^{* * *} \\
(0.015)\end{array}$ \\
\hline Financial openness & & & $\begin{array}{l}-2.938 \\
(3.420)\end{array}$ & & & $\begin{array}{l}-1.258 \\
(1.934)\end{array}$ \\
\hline Banking supervision & & & $\begin{array}{l}-0.539 \\
(1.335)\end{array}$ & & & $\begin{array}{l}-0.153 \\
(0.605)\end{array}$ \\
\hline Constant & $\begin{array}{c}15.238^{* * *} \\
(1.840)\end{array}$ & $\begin{array}{c}13.703^{* * *} \\
(2.325)\end{array}$ & $\begin{array}{c}21.073^{* * *} \\
(6.918) \\
\end{array}$ & $\begin{array}{c}12.779^{* * *} \\
(1.155)\end{array}$ & $\begin{array}{c}10.493^{* * *} \\
(1.280)\end{array}$ & $\begin{array}{c}11.969^{* * *} \\
(3.222)\end{array}$ \\
\hline Observations & 738 & 641 & 343 & 457 & 429 & 187 \\
\hline R-squared & 0.041 & 0.053 & 0.058 & 0.115 & 0.167 & 0.161 \\
\hline Number of countries & 70 & 66 & 53 & 54 & 52 & 40 \\
\hline CONS_W & $\begin{array}{c}-3.230^{* * *} \\
(1.082)\end{array}$ & $\begin{array}{c}-3.383^{* * *} \\
(1.208)\end{array}$ & $\begin{array}{c}-4.662^{* *} \\
(2.265)\end{array}$ & $\begin{array}{c}-3.096^{* * *} \\
(0.614)\end{array}$ & $\begin{array}{c}-2.014^{* * *} \\
(0.608)\end{array}$ & $\begin{array}{l}-1.043 \\
(1.157)\end{array}$ \\
\hline Variance of supply shocks & $\begin{array}{c}0.244 \\
(0.465)\end{array}$ & $\begin{array}{c}-0.063 \\
(0.516)\end{array}$ & $\begin{array}{l}-0.858 \\
(0.961)\end{array}$ & $\begin{array}{c}-0.008 \\
(0.218)\end{array}$ & $\begin{array}{c}-0.073 \\
(0.231)\end{array}$ & $\begin{array}{c}-0.719^{*} \\
(0.398)\end{array}$ \\
\hline Variance of demand shocks & $\begin{array}{c}-1.181^{* * *} \\
(0.416)\end{array}$ & $\begin{array}{c}-1.217^{* * *} \\
(0.444)\end{array}$ & $\begin{array}{c}-2.031^{* *} \\
(0.944)\end{array}$ & $\begin{array}{l}-0.151 \\
(0.209)\end{array}$ & $\begin{array}{c}-0.429^{* *} \\
(0.202)\end{array}$ & $\begin{array}{l}-0.576 \\
(0.410)\end{array}$ \\
\hline GDP per capita & $\begin{array}{l}-0.015 \\
(0.013)\end{array}$ & $\begin{array}{c}-0.024 \\
(0.015)\end{array}$ & $\begin{array}{l}-0.014 \\
(0.032)\end{array}$ & $\begin{array}{c}-0.014^{*} \\
(0.008)\end{array}$ & $\begin{array}{c}-0.017^{* *} \\
(0.008)\end{array}$ & $\begin{array}{l}-0.027 \\
(0.018)\end{array}$ \\
\hline Lerner index & & $\begin{array}{c}2.383 \\
(2.040)\end{array}$ & $\begin{array}{l}-2.020 \\
(3.491)\end{array}$ & & $\begin{array}{c}3.776^{* * *} \\
(0.962)\end{array}$ & $\begin{array}{l}2.664^{*} \\
(1.511)\end{array}$ \\
\hline Bank concentration & & $\begin{array}{c}0.014 \\
(0.017)\end{array}$ & $\begin{array}{l}-0.009 \\
(0.029)\end{array}$ & & $\begin{array}{c}0.025^{* * *} \\
(0.008)\end{array}$ & $\begin{array}{c}0.043^{* * *} \\
(0.015)\end{array}$ \\
\hline Financial openness & & & $\begin{array}{l}-2.934 \\
(3.417)\end{array}$ & & & $\begin{array}{l}-1.329 \\
(1.958)\end{array}$ \\
\hline Banking supervision & & & $\begin{array}{c}-0.607 \\
(1.332)\end{array}$ & & & $\begin{array}{c}-0.183 \\
(0.606)\end{array}$ \\
\hline Constant & $\begin{array}{c}15.833^{* * *} \\
(1.824)\end{array}$ & $\begin{array}{c}14.907^{* * *} \\
(2.348)\end{array}$ & $\begin{array}{c}21.327^{* * *} \\
(6.920)\end{array}$ & $\begin{array}{c}12.909^{* * *} \\
(1.159)\end{array}$ & $\begin{array}{c}10.636^{* * *} \\
(1.307)\end{array}$ & $\begin{array}{c}12.066^{* * *} \\
(3.318)\end{array}$ \\
\hline Observations & 738 & 641 & 343 & 457 & 429 & 187 \\
\hline R-squared & 0.048 & 0.061 & 0.060 & 0.117 & 0.165 & 0.159 \\
\hline Number of countries & 70 & 66 & 53 & 54 & 52 & 40 \\
\hline
\end{tabular}

Note: Standard errors are reported in parentheses. $*$; $* *$, and $* * *$ indicate statistical significance at the $10 \%, 5 \%$ and $1 \%$ level, respectively. 
Due to data availability, it is important to note that the three specifications that we consider cover different time periods. The specification (1) covers the period from 1980 to 2012. The specification (2) covers the period from 1996 to 2012, as the competition and concentration measures provided by GFD database are available since 1996 . Finally, the specification (3), which includes all control variables, covers the period from 1996 to 2005, because the measure of banking sector supervision provided by Abiad et al. (2010) are only available until $2005^{13}$.

For all the specifications reported from Tables 1 to 3, we find a robust relationship between the measure of inflation aversion of the central bank and the level of banking sector vulnerability. Except specification (3), with the capital-to-asset ratio as the endogenous variable, the coefficients associated with the CONS index are significant at the conventional levels, with a sign that validates the benign neglect hypothesis. This also applies to the CONS_W index. The link between the central banks' inflation aversion and the vulnerability of the banking sector is even stronger with $C O N S \_W$, in particular with the the Z-scrore as dependent variable. Thus, the more the central banks focus on the inflation goal, the more this increases fragility of the banking sector. Credit cycles are amplified, with more excessive and volatile amounts of credit (Table 1) and banks' balance sheets are deteriorated (Tables 2 and 3). A higher degree of $\mathrm{CBC}$ clearly entails higher banking sector vulnerability. Importantly, this result holds despite changes in the sample size and the period covered due to data availability, in particular once variables capturing the banking market structure and financial regulation are included. This is a first evidence of robustness.

The non-significance of the coefficient for the central banks' preferences when the capital-to-asset ratio is used as the dependent variable in specification (3) can easily be explained. Since the late 1980s, the Basel Committee on Banking Supervision (BCBS) has made recommendations on regulations on bank capital and leverage. The most striking example is the implementation in 1992 of the Cook ratio as an international norm for banks' capital. Such requirements were followed by many countries whatever the preferences of their central banks. In our sample, no country has an average capital-to-asset ratio below the reference value of $3 \%^{14}$ (the norm recommended by the Basel III agreement, see BIS, 2014). This is the case for the 40 countries that remain once financial openness and regulation data are considered in specification (3). In consequence, this variable does not act as a discriminant indicator of banking sector vulnerability for these countries.

Moreover, the significance of the control variables depends on both the sample size and the choice of the dependent variable, particularly for macroeconomic shocks. As highlighted above, the expected sign of banking competition is unclear. When the Lerner coefficient is significant, competition between banks tends to weaken the banking sector in most cases. Our result highlights the "competition-fragility" view mentioned above. This explanation is particularly relevant when we consider the non-

\footnotetext{
${ }^{13}$ Unfortunately, there does not yet exist in the literature a database on banking sector supervision and regulation that covers a longer time span. Due to data availability, please also note that, for each specification, the time period depends on the endogenous variable considered. The credit volatility, the credit-to-GDP gap, and the credit-to-deposit ratio cover the period from 1980 to 2012, while the other measures of banking sector vulnerability cover the period 1998 to 2012.

${ }^{14}$ In the measure we use, the definition of banks' capital is broader than those adopted by the Basel Committee; however, the measure also underestimates banks' assets because, unlike the Basel III agreement, it does not consider off-balance-sheet assets. Therefore, the $3 \%$ threshold can be considered more restrictive for our measure.
} 
performing loans ratio, the capital-to-asset ratio, and the credit volatility as endogenous variables. The coefficients associated with the concentration index lead to the same conclusion with the credit-to-deposit ratio and the capital-to-asset ratio as lefthand side variables. A more concentrated banking market seems to lead to a more stable financial sector. Next, the coefficient estimates associated with the banking sector supervision appear not statistically significant at the conventional levels. Finally, financial openness is only significant when we consider the credit-to-GDP gap as an endogenous variable. Overall, the signs associated with the control variables are consistent with the theoretical arguments raised in the literature.

\section{Robustness checks}

To enhance the credibility and plausibility of our earlier findings, we check the robustness of our baseline results in five ways. First, we address the fact that the global shocks that emerging and industrialised countries face are not necessarily symmetric, and we also control for the potential break induced by the subprime crisis. Second, we check whether our baseline results are sensitive to the set of control variables by considering some alternative control variables. Third, we perform the same exercise by considering additional right-hand side variables capturing some features of the monetary policy framework and characteristics of the banking sector. Fourth, we propose an alternative measure of central banks' preferences which explicitly takes into account some other factors that could impact the inflation and output gap volatility. Finally, we address the potential reverse causality issue between central banks' preferences and the vulnerability of the banking sector by re-estimating our baseline model using a two-stage least squares (2SLS) estimator.

Sample heterogeneity and subprime crisis. The recent evidence suggests that industrialised, emerging, and developing countries have not been impacted in a similar way by the subprime crisis. Indeed, a number of developing economies were protected from the immediate consequences of the financial crisis by relatively underdeveloped financial markets and limited international linkages. Instead, they were impacted by the crisis more gradually and through somewhat different channels than developed and emerging countries, such as the decline of global trade, the decline of remittances, and reduced capital flows.

Given potential heterogeneity, we check the robustness of our results in two ways. First, we replace time fixed effects by time-group fixed effects, by considering two groups of countries, OECD and non-OECD. By this way, we take explicitly into account the fact that common shocks are not the same for OECD and non-OECD countries. Results that we obtain concerning the CONS and CONS_W indexes are not impacted by this change ${ }^{15}$.

Second, we include a dummy variable capturing the subprime crisis. However, since the banking sectors of economies around the world have not been impacted in the same way by the subprime crisis, we do not consider the same dummy variable for all countries of our sample. As our main research question concerns the fragility of the banking sector, we prefer to consider a subprime crisis dummy variable equal to 1 for countries which have known at the same period a systemic banking crisis, and 0

\footnotetext{
${ }^{15}$ Results are available upon request.
} 
otherwise. To this end, we refer to the comprehensive database on systemic banking crises compiled by Laeven and Valencia $(2013)^{16}$. Results that we obtain are reported in Table 4. To save space, we only report the coefficient estimates associated with the $C O N S$ and $C O N S \_W$ indexes, and those associated with the subprime crisis dummy variable ${ }^{17}$.

As expected, for both specifications, we find a robust and significant positive relationship between the crisis dummy and the credit volatility. This result is closely linked to the slowdown in credit growth in the aftermath of the crisis, which exacerbates the volatility of credit $^{18}$. This decline in bank lending probably also explains the negative relationship that we observe between the crisis dummy and the credit-to-deposit ratio, even if this relationship is only statistically significant for the specification (1). Similar results are obtained for the nonperforming loans ratio and the Z-score. More importantly, we still observe a significant relationship between the CONS (or CONS_W) index and our alternative measures of banking sector vulnerability. This confirms our initial findings that central banks' preferences are an important driver of the fragility of the banking sector.

\footnotetext{
${ }^{16}$ More precisely, if a country has known a systemic banking crisis during the recent financial turmoil, we consider a dummy variable equal to 1 until 2012, while the starting date depends on the information provided by Laeven and Valencia (2013).

${ }^{17}$ The specification (3) is not considered because, as explained above, it only covers the period from 1996 to 2005.

${ }^{18}$ Indeed, this variable is calculated as a five-year moving variance on quarterly credit data.
} 
Table 4: Robustness checks - Results obtained with a subprime crisis dummy as additional control variable

\begin{tabular}{|c|c|c|c|c|c|c|}
\hline & \multicolumn{6}{|c|}{ Results obtained with CONS index } \\
\hline \multirow[t]{2}{*}{ Dependent variable } & \multicolumn{2}{|c|}{ Credit volatility } & \multicolumn{2}{|c|}{ Credit-to-GDP gap } & \multicolumn{2}{|c|}{ Credit-to-deposit ratio } \\
\hline & (1) & $(2)$ & & $(2)$ & (1) & $(2)$ \\
\hline \multirow[t]{2}{*}{ CONS } & $19.890^{*}$ & $59.062^{* * *}$ & $15.753^{* * *}$ & $17.320^{* * *}$ & $20.107^{* * *}$ & $31.832^{* * *}$ \\
\hline & $(11.009)$ & $(20.246)$ & $(2.727)$ & $(3.580)$ & $(5.442)$ & $(6.668)$ \\
\hline \multirow[t]{4}{*}{ Subprime crisis dummy } & $33.374^{* *}$ & $32.207^{*}$ & -4.525 & -0.411 & $-18.938^{* * *}$ & -2.784 \\
\hline & $(14.102)$ & $(18.949)$ & $(2.778)$ & $(2.874)$ & $(6.484)$ & $(6.326)$ \\
\hline & Nonperfor & g loans ratio & & & Capital-to & sset ratio \\
\hline & (1) & $(2)$ & $(1)$ & $(2)$ & (1) & $(2)$ \\
\hline \multirow[t]{2}{*}{ CONS } & $6.234^{* * *}$ & $6.552^{* * *}$ & $-1.998^{*}$ & -1.840 & $-3.033^{* * *}$ & $-2.019 * * *$ \\
\hline & $(1.385)$ & $(1.430)$ & $(1.115)$ & $(1.215)$ & $(0.603)$ & $(0.587)$ \\
\hline \multirow[t]{5}{*}{ Subprime crisis dummy } & $1.824^{*}$ & 0.727 & $-1.609^{*}$ & -1.096 & 0.507 & 0.116 \\
\hline & $(1.020)$ & $(1.056)$ & $(0.931)$ & $(0.971)$ & $(0.415)$ & $(0.409)$ \\
\hline & \multicolumn{6}{|c|}{ Results obtained with CONS_W index } \\
\hline & \multicolumn{2}{|c|}{ Credit volatility } & \multicolumn{2}{|c|}{ Credit-to-GDP gap } & \multicolumn{2}{|c|}{ Credit-to-deposit ratio } \\
\hline & (1) & $(2)$ & (1) & $(2)$ & (1) & $(2)$ \\
\hline \multirow[t]{2}{*}{ CONS_W } & $25.967^{* *}$ & $63.839^{* * *}$ & $12.946^{* * *}$ & $15.357^{* * *}$ & $14.576^{* * *}$ & $25.867^{* * *}$ \\
\hline & $(10.748)$ & $(20.423)$ & $(2.690)$ & $(3.618)$ & $(5.353)$ & $(6.743)$ \\
\hline \multirow[t]{4}{*}{ Subprime crisis dummy } & $33.418^{* *}$ & $34.090^{*}$ & -3.977 & 0.293 & $-18.510^{* * *}$ & -1.991 \\
\hline & $(14.060)$ & $(18.872)$ & $(2.788)$ & $(2.873)$ & $(6.509)$ & $(6.373)$ \\
\hline & Nonperfor & ag loans ratio & & & Capital-to & sset ratio \\
\hline & (1) & $(2)$ & (1) & $(2)$ & (1) & $(2)$ \\
\hline \multirow[t]{2}{*}{ CONS_W } & $6.055^{* * *}$ & $5.819^{* * *}$ & $-3.009^{* * *}$ & $-3.223^{* * *}$ & $-3.163^{* * *}$ & $-2.020 * * *$ \\
\hline & $(1.412)$ & $(1.469)$ & $(1.088)$ & $(1.217)$ & $(0.617)$ & $(0.612)$ \\
\hline \multirow[t]{2}{*}{ Subprime crisis dummy } & $1.948^{*}$ & 0.977 & $-1.535^{*}$ & -1.015 & 0.446 & 0.037 \\
\hline & $(1.019)$ & $(1.056)$ & $(0.925)$ & $(0.962)$ & $(0.413)$ & $(0.407)$ \\
\hline
\end{tabular}

Note: Standard errors are reported in parentheses. *; **, and *** indicate statistical significance at the $10 \%, 5 \%$ and $1 \%$ level, respectively.

Alternative control variables. We assess whether our baseline results are sensitive to the set of control variables by considering some alternative control variables. First, following Demirgüç-Kunt and Detragiache (1998), we replace demand and supply shocks with the annual growth rate of real GDP and the annual inflation rate. These two variables are taken from the WDI database and constitute an alternative approach to capturing macroeconomic shocks that may adversely affect the economy and the banking system and, in turn, drive financial imbalances.

Second, we consider two alternative proxies for banking competition. We replace the Lerner index with the Boone index. As the Lerner index, the Boone index is a non-structural competition measure and is taken from the GFD database. Despite the intensive academic debate between the proponents of the Lerner index and those of the Boone index, some recent empirical papers in the banking literature use the Boone index as a measure of bank competition (see, e.g. Schaeck and Čihák, 2014; Van Leuvensteijn et al., 2011). In the same way, we consider an alternative measure of bank concentration, defined as the assets of the five largest commercial banks, rather than the three largest, as a share of total assets of the banking sector ${ }^{19}$.

Third, we re-estimate our baseline model by replacing the banking supervision

\footnotetext{
${ }^{19}$ Please also note that our main empirical findings are not affected when we re-estimate our model by considering individually each of the four bank competition proxies considered in this paper.
} 
index with a measure of de jure supervisory power to give a more complete picture of prudential regulation. This index has been developed by Barth et al. (2004) and lies between 0 and 16. The expected sign of the variable is negative, as a higher value implies greater supervisory power.

Finally, we consider a proxy for the quality of domestic institutions as an alternative to the banking supervision index. This choice is driven by several considerations. As argued by Demirgüç-Kunt and Detragiache (1998), the quality of domestic institutions is highly related to the ability of the government to implement effective prudential supervision. Moreover, a weak institutional framework is expected to exacerbate financial fragility, as it provides limited judicial protection to creditors and shareholders (Shimpalee and Breuer, 2006). Given this, we use the "Law and order" index compiled by the International Country Risk Guide (ICRG). This index lies between 0 and 6 , with a higher value indicating better institutional quality. It has been widely used in the empirical literature devoted to financial fragility (see, e.g. Kaminsky and Schmukler, 2003; Demirgüç-Kunt and Detragiache, 1998).

Results that we obtain, available upon request, suggest that considering alternative control variables does not affect our main results. Indeed, we still find a statistically significant relationship between central banks' preferences and our different variables proxying the vulnerability of the banking sector. As for our baseline estimates, we find a positive and significant relationship between the CONS (or CONS_W) index and the credit volatility, the credit-to-GDP gap, the credit-to-deposit ratio, and the nonperforming loans ratio, and a negative and significant relationship between the $C O N S$ (or CONS_W) index and the Z-score, and the capital-to-asset ratio. Then, our findings confirm that strong preferences of the central bank for price stability exacerbate the vulnerability of the banking sector, and then financial instability.

Controlling for the features of the monetary policy framework. We extend the set of control variables by considering three different features of the monetary policy framework: the degree of central bank independence, the type of exchange rate regime and, whether a central bank pursues or not an inflation targeting strategy.

According to Čihák (2007), the independence of a central bank could enhance financial stability. Indeed, independence from the political authorities reduces monetary policy constraint and allows central bankers to react quicker and stronger to financial distress. On the contrary, dependent central banks delay their response to financial imbalances, as electoral purposes lead policymakers to maintain the status quo in order to transfer the problem to their successor. The empirical analysis conducted by Klomp and de Haan (2009) supports this view. Using bank-level data, Doumpos et al. (2015) reach the same conclusion. They show that the independence of the central bank and other supervisory agencies exercises a positive impact on the soundness of banking institutions. Nevertheless, some other papers support the opposite view (see, e.g. Aklin and Kern, 2016; Berger and Kißmer, 2013). Furthermore, other studies focus on the role of inflation targeting framework on financial stability (see, e.g. Fazio et al., 2015; Frappa and Mésonnier, 2010). However, these papers reach opposite conclusions. The last important feature of the monetary policy framework that we consider is the exchange rate regime. Indeed, a number of empirical studies investigate the linkages between exchange rate regimes and banking crises (see, e.g. Domaç and Martinez Peria, 2003).

The degree of central bank independence is measured using two well-known proxies 
introduced by Cukierman et al. (1992): the CWN index and the turnover rate of central bank governors (TOR index). The former is a de jure index of central bank independence. The CWN index has been recently updated by Garriga (2016) for a large sample of countries. The TOR index is commonly used in the literature as an inverse proxy for central bank independence. It is viewed as more reliable when the rule of law is not strongly embedded in the political culture, as is sometimes the case in some developing and emerging countries. The index is computed over five-year rolling windows, and information on the term in office of central bank governors comes from Dreher et al. (2008). Consequently, as our sample contains advanced and emerging countries, we include simultaneously these two proxies for central bank independence as additional control variables.

As usual in the literature, we consider two alternative binary variables for controlling for the adoption of an inflation targeting framework. The first takes the value of 1 when a country in a giving year has at least partially adopted inflation targeting as a monetary policy strategy, and zero otherwise. The second measures the fully-fledged adoption of an inflation targeting framework. It takes the value of 1 only when an inflation targeting country satisfies all prerequisites for inflation targeting, and zero otherwise.

Finally, to control for the exchange rate regime, we use a de jure and a de facto classification. Both classifications are polynomial variables and come from the classification taxonomy introduced by the International Monetary Fund (IMF) and Ghosh et al. (2011). Exchange rate regimes are classified into eight categories, from the less flexible regime (1) to the most flexible (8).

We re-estimate our three baseline specifications by including individually each of the additional control variables discussed above. Results that we obtain are reported in Tables 5 to 7 . To save space, we only report the coefficient estimates for the CONS and $C O N S \_W$ indexes. As we can see, results are quite similar to our initial findings and thus confirm the robustness of the link between central banks' preferences and banking sector vulnerability. Concerning the additional control variables, in line with the existing empirical literature, results that we obtain are more mixed ${ }^{20}$.

Controlling for other characteristics of the banking sector. We extend the set of control variables by considering different features of the banking industry. First, following the recent empirical literature having investigated the competition-efficiencystability nexus, we consider additional control variables proxying the efficiency of the banking sector. Indeed, as shown by Schaeck and Čihák (2014), efficiency is the transmission mechanism through which competition contributes to stability. The proxies for banking sector efficiency that we consider are the bank cost to income ratio and the bank overhead costs to total assets ratio. They are taken form the GFD database. The bank cost to income ratio is defined as the operating expenses of a bank as a share of sum of net-interest revenue and other operating income, while the bank overhead costs to total assets ratio represents the operating expenses of a bank as a share of the value of all assets held. These two variables are expressed in percentage terms. The World Bank computes them by considering for each country the median of the

\footnotetext{
${ }^{20}$ We have also tested the potential indirect effects of these features of the monetary policy framework through interaction terms with our two measures of central banks' preferences, namely the CONS and CONS_W indexes. Results that we obtain suggest that interaction terms are generally not statistically significant at the conventional levels.
} 
bank-level ratios.

Second, we control for the diversification of income sources in the banking industry, i.e. interest $v s$ non-interest income ${ }^{21}$. Indeed, the link between the income diversification and bank risk has attracted increasing attention in academic research ${ }^{22}$. Our measure of income diversification is taken from the GFD database, and is defined as the ratio of bank non-interest income to total income. As for the measures of banking sector efficiency, the World Bank considers for each country the median of the bank-level ratios.

Third, we control for the relative importance of the foreign bank presence in domestic banking systems. As well-documented by Claessens and Van Horen (2014), there is an extensive debate in the academic literature concerning the potential costs and benefits of foreign bank ownership, particularly in terms of financial stability. The variable that we consider to measure the relative importance of the foreign bank presence is taken from the GFD database, and corresponds to the percentage of the number of foreign owned banks to the number of the total banks in the host economy. A bank is defined as foreign-owned if $50 \%$ or more of its shares are owned by foreigners ${ }^{23}$.

Fourth, we take into account the fact that risk may be different for governmentowned banks and privately-owned banks, and then, the fact that the ownership structure of the banking sector may impact its vulnerability (see, e.g. Barth et al., 2004; La Porta et al., 2002). Following the existing macro literature, we measure the relative importance of government ownership in the banking industry as the share of stateowned or state controlled bank assets on the total banking sector assets. A bank is classified as state-owned when the government's equity ownership exceeds $50 \%$. This variable is taken from Barth et al. (2004), and covers the period 1999-2012 ${ }^{24}$.

Finally, we test the robustness of our results by including the capital flows as an additional control variable in the specification (3). Following Calvo et al. (2008), the measure of capital flows is calculated as the sum of foreign direct and portfolio investments, using data constructed by Lane and Milesi-Ferretti (2007). While we used the Chinn-Ito index as a proxy for legal financial openness in our baseline estimates, this test accounts simultaneously for both the legal and the actual dimensions of financial openness.

Results that we obtain when we consider these additional control variables are reported Tables 5 to 7 . To save space, we only report the coefficient estimates for the CONS and CONS_W indexes. We can see that our findings still support a strong relationship between central banks' preferences for inflation stabilization and the fragility of the banking sector.

\footnotetext{
${ }^{21}$ Non-interest income includes in particular income from trading and securitization, investment banking and advisory fees, brokerage commissions, venture capital, fiduciary income, and gains on non-hedging derivatives.

${ }^{22}$ See, e.g. Köhler (2015) and DeYoung and Roland (2001).

${ }^{23}$ The Global Financial Development Database (GFDD) also provides as a measure of foreign bank presence the foreign bank assets to total bank assets ratio. Unfortunately, this variable is only available since 2005.

${ }^{24}$ More precisely, Barth et al. (2004) conducted four surveys on bank regulation (1999, 2003, 2007, and 2011). To conserve the panel structure of our data, we consider the results of the 1st survey for the years 1999-2002, of the 2nd survey for the years 2003-2006, of the 3rd for the years 2007-2010 and, years 2011 and 2012 correspond to the results of the 4 th survey.
} 
Table 5: Robustness checks - Additional control variables: Results obtained with the credit volatility and the credit-to-GDP gap

\begin{tabular}{|c|c|c|c|c|c|c|}
\hline \multirow{3}{*}{$\begin{array}{l}\text { Measure of central banks' preferences } \\
\text { Dependent variable }\end{array}$} & \multicolumn{6}{|c|}{ CONS } \\
\hline & \multicolumn{3}{|c|}{ Credit volatility } & \multicolumn{3}{|c|}{ Credit-to-GDP gap } \\
\hline & (1) & $(2)$ & (3) & (1) & $(2)$ & (3) \\
\hline \multicolumn{7}{|l|}{ Additional control variable } \\
\hline Central bank independence & $\begin{array}{l}21.049^{*} \\
(11.716)\end{array}$ & $\begin{array}{c}71.885^{* * *} \\
(21.918)\end{array}$ & $\begin{array}{c}51.727^{* * *} \\
(13.840)\end{array}$ & $\begin{array}{c}16.310^{* * *} \\
(2.809)\end{array}$ & $\begin{array}{c}16.886^{* * *} \\
(3.627)\end{array}$ & $\begin{array}{c}13.121^{* *} \\
(5.719)\end{array}$ \\
\hline Partial adoption of inflation targeting & $\begin{array}{c}21.913^{* *} \\
(11.020)\end{array}$ & $\begin{array}{c}62.473^{* * *} \\
(20.372)\end{array}$ & $\begin{array}{c}46.323^{* * *} \\
(13.287)\end{array}$ & $\begin{array}{c}15.371^{* * *} \\
(2.710)\end{array}$ & $\begin{array}{c}17.443^{* * *} \\
(3.556)\end{array}$ & $\begin{array}{c}14.602^{* *} \\
(5.639)\end{array}$ \\
\hline Fully-fledged inflation targeting & $\begin{array}{c}22.209^{* *} \\
(11.013)\end{array}$ & $\begin{array}{c}61.855^{* * *} \\
(20.311)\end{array}$ & $\begin{array}{c}45.320^{* * *} \\
(13.203)\end{array}$ & $\begin{array}{c}15.366^{* * *} \\
(2.710)\end{array}$ & $\begin{array}{c}17.132^{* * *} \\
(3.552)\end{array}$ & $\begin{array}{c}14.694^{* * *} \\
(5.583)\end{array}$ \\
\hline De jure exchange rate regime & $\begin{array}{c}21.983^{* *} \\
(11.001)\end{array}$ & $\begin{array}{c}73.149^{* * *} \\
(21.602)\end{array}$ & $\begin{array}{c}49.130^{* * *} \\
(13.659)\end{array}$ & $\begin{array}{c}13.115^{* * * *} \\
(3.258)\end{array}$ & $\begin{array}{c}14.917^{* * *} \\
(4.909)\end{array}$ & $\begin{array}{c}15.415^{* * *} \\
(5.804)\end{array}$ \\
\hline De facto exchange rate regime & $\begin{array}{l}20.250^{*} \\
(10.824)\end{array}$ & $\begin{array}{c}68.075^{* * *} \\
(20.763)\end{array}$ & $\begin{array}{c}46.375^{* * *} \\
(13.206)\end{array}$ & $\begin{array}{c}13.781^{* * * *} \\
(3.215)\end{array}$ & $\begin{array}{c}15.441^{* * *} \\
(4.773)\end{array}$ & $\begin{array}{c}14.489^{* *} \\
(5.607)\end{array}$ \\
\hline Bank cost-to-income ratio & & $\begin{array}{c}62.033^{* * * *} \\
(20.260)\end{array}$ & $\begin{array}{c}46.134^{* * * *} \\
(13.269)\end{array}$ & & $\begin{array}{c}17.198^{* * * *} \\
(3.539)\end{array}$ & $\begin{array}{c}14.780^{* * * *} \\
(5.623)\end{array}$ \\
\hline Bank overhead costs & & $\begin{array}{c}60.336^{* * * *} \\
(20.557)\end{array}$ & $\begin{array}{c}42.607^{* * * *} \\
(13.339)\end{array}$ & & $\begin{array}{c}17.602^{* * * *} \\
(3.527)\end{array}$ & $\begin{array}{c}13.158^{* *} \\
(5.640)\end{array}$ \\
\hline Income diversification & & $\begin{array}{c}56.790^{* * *} \\
(20.245)\end{array}$ & $\begin{array}{c}44.228^{* * *} \\
(13.250)\end{array}$ & & $\begin{array}{c}17.114^{* * *} \\
(3.569)\end{array}$ & $\begin{array}{c}15.913^{* * * *} \\
(5.588)\end{array}$ \\
\hline Foreign bank presence & & $\begin{array}{c}64.359^{* * *} \\
(20.392)\end{array}$ & $\begin{array}{c}48.602^{* * *} \\
(13.305)\end{array}$ & & $\begin{array}{c}15.814^{* * *} \\
(3.515)\end{array}$ & $\begin{array}{c}13.785^{* *} \\
(5.655)\end{array}$ \\
\hline State-owned bank presence & & $\begin{array}{c}103.431^{* * *} \\
(30.228)\end{array}$ & $\begin{array}{c}86.452^{* * *} \\
(21.866)\end{array}$ & & $\begin{array}{c}13.848^{* * *} \\
(3.738)\end{array}$ & $\begin{array}{c}15.849^{* * *} \\
(6.020)\end{array}$ \\
\hline De facto financial openness & & & $\begin{array}{c}46.349^{* * *} \\
(13.326)\end{array}$ & & & $\begin{array}{c}15.279^{* * *} \\
(5.620)\end{array}$ \\
\hline Measure of central banks' preferences & \multicolumn{6}{|c|}{ CONS_W } \\
\hline Dependent variable & \multirow{2}{*}{\multicolumn{3}{|c|}{ Credit volatility }} & \multicolumn{3}{|c|}{ Credit-to-GDP gap } \\
\hline & & & & $(1)$ & $(2)$ & (3) \\
\hline Additional control variable & & & & & & \\
\hline Central banks independence & $\begin{array}{c}27.261^{* *} \\
(11.419)\end{array}$ & $\begin{array}{c}78.734^{* * *} \\
(22.441)\end{array}$ & $\begin{array}{c}52.386^{* * *} \\
(13.754)\end{array}$ & $\begin{array}{c}13.468^{* * *} \\
(2.761)\end{array}$ & $\begin{array}{c}15.332^{* * *} \\
(3.713)\end{array}$ & $\begin{array}{c}13.831^{* *} \\
(5.677)\end{array}$ \\
\hline Partial adoption of inflation targeting & $\begin{array}{c}27.451^{* *} \\
(10.776)\end{array}$ & $\begin{array}{c}65.905^{* * *} \\
(20.563)\end{array}$ & $\begin{array}{c}45.779^{* * *} \\
(13.098)\end{array}$ & $\begin{array}{c}12.816^{\text {*** }} \\
(2.678)\end{array}$ & $\begin{array}{c}15.495^{* * *} \\
(3.603)\end{array}$ & $\begin{array}{c}15.204^{\text {*** }} \\
(5.544)\end{array}$ \\
\hline Fully-fledged inflation targeting & $\begin{array}{c}27.782^{\text {*** }} \\
(10.768)\end{array}$ & $\begin{array}{c}65.480^{* * *} \\
(20.492)\end{array}$ & $\begin{array}{c}45.102^{* * *} \\
(13.037)\end{array}$ & $\begin{array}{c}12.800^{* * *} \\
(2.677)\end{array}$ & $\begin{array}{c}15.298^{* * *} \\
(3.596)\end{array}$ & $\begin{array}{c}15.581^{* * *} \\
(5.497)\end{array}$ \\
\hline De jure exchange rate regime & $\begin{array}{c}26.444^{* *} \\
(10.677)\end{array}$ & $\begin{array}{c}72.358^{* * *} \\
(21.525)\end{array}$ & $\begin{array}{c}48.291^{* * *} \\
(13.426)\end{array}$ & $\begin{array}{c}11.270^{* * * *} \\
(3.186)\end{array}$ & $\begin{array}{c}14.541^{* * *} \\
(4.888)\end{array}$ & $\begin{array}{c}15.925^{* * *} \\
(5.690)\end{array}$ \\
\hline De facto exchange rate regime & $\begin{array}{c}24.894^{* *} \\
(10.494)\end{array}$ & $\begin{array}{c}68.350^{* * * *} \\
(20.781)\end{array}$ & $\begin{array}{c}45.712^{* * * *} \\
(13.028)\end{array}$ & $\begin{array}{c}12.037^{* * *} \\
(3.141)\end{array}$ & $\begin{array}{c}15.117^{* * * *} \\
(4.776)\end{array}$ & $\begin{array}{c}15.161^{* * * *} \\
(5.515)\end{array}$ \\
\hline Bank cost-to-income ratio & & $\begin{array}{c}65.544^{* * *} \\
(20.506)\end{array}$ & $\begin{array}{c}45.535^{* * *} \\
(13.095)\end{array}$ & & $\begin{array}{c}15.297^{* * *} \\
(3.594)\end{array}$ & $\begin{array}{c}15.226^{* * *} \\
(5.535)\end{array}$ \\
\hline Bank overhead costs & & $\begin{array}{c}64.009 * * * \\
(20.772)\end{array}$ & $\begin{array}{c}42.236^{* * *} \\
(13.199)\end{array}$ & & $\begin{array}{c}15.867^{* * *} \\
(3.584)\end{array}$ & $\begin{array}{c}13.704^{* *} \\
(5.567)\end{array}$ \\
\hline Income diversification & & $\begin{array}{c}62.358^{* * *} \\
(20.394)\end{array}$ & $\begin{array}{c}43.987^{* * * *} \\
(13.056)\end{array}$ & & $\begin{array}{c}15.162^{* * *} \\
(3.604)\end{array}$ & $\begin{array}{c}16.097^{* * *} \\
(5.498)\end{array}$ \\
\hline Foreign bank presence & & $\begin{array}{c}67.627^{* * *} \\
(20.592)\end{array}$ & $\begin{array}{c}47.258^{* * *} \\
(13.080)\end{array}$ & & $\begin{array}{c}14.456^{* * *} \\
(3.551)\end{array}$ & $\begin{array}{c}14.583^{* * *} \\
(5.540)\end{array}$ \\
\hline State-owned bank presence & & $\begin{array}{c}111.780^{* * * *} \\
(31.291)\end{array}$ & $\begin{array}{c}89.860^{* * *} \\
(22.360)\end{array}$ & & $\begin{array}{c}14.278^{* * *} \\
(3.844)\end{array}$ & $\begin{array}{c}18.650^{* * *} \\
(6.124)\end{array}$ \\
\hline De facto financial openness & & & $\begin{array}{c}46.335^{* * *} \\
(13.209)\end{array}$ & & & $\begin{array}{c}15.692^{* * *} \\
(5.560)\end{array}$ \\
\hline
\end{tabular}

Note: Standard errors are reported in parentheses. ${ }^{*} ; * *$, and $* * *$ indicate statistical significance at the $10 \%, 5 \%$ and $1 \%$ level, respectively. 
Table 6: Robustness checks - Additional control variables: Results obtained with the credit-to-deposit ratio and the nonperforming loans ratio

\begin{tabular}{|c|c|c|c|c|c|c|}
\hline \multirow{3}{*}{$\begin{array}{l}\text { Measure of central banks' preferences } \\
\text { Dependent variable }\end{array}$} & \multicolumn{6}{|c|}{ CONS } \\
\hline & \multicolumn{3}{|c|}{ Credit-to-deposit ratio } & \multicolumn{3}{|c|}{ Nonperforming loans ratio } \\
\hline & (1) & $(2)$ & $(3)$ & $(1)$ & $(2)$ & $(3)$ \\
\hline Additional control variable & & & & & & \\
\hline Central bank independence & $\begin{array}{c}19.753^{* * *} \\
(5.685)\end{array}$ & $\begin{array}{c}31.555^{* * *} \\
(6.813)\end{array}$ & $\begin{array}{l}21.648^{*} \\
(11.116)\end{array}$ & $\begin{array}{c}5.755^{* * *} \\
(1.224)\end{array}$ & $\begin{array}{c}6.273^{* * *} \\
(1.252)\end{array}$ & $\begin{array}{l}4.729^{* *} \\
(1.978)\end{array}$ \\
\hline Partial adoption of inflation targeting & $\begin{array}{c}19.424^{* * *} \\
(5.443)\end{array}$ & $\begin{array}{c}31.536^{* * *} \\
(6.649)\end{array}$ & $\begin{array}{c}23.129^{* *} \\
(10.764)\end{array}$ & $\begin{array}{c}6.278^{* * *} \\
(1.378)\end{array}$ & $\begin{array}{c}6.423^{* * *} \\
(1.419)\end{array}$ & $\begin{array}{l}4.618^{* *} \\
(1.879)\end{array}$ \\
\hline Fully-fledged inflation targeting & $\begin{array}{c}19.585^{* * *} \\
(5.440)\end{array}$ & $\begin{array}{c}31.347^{* * *} \\
(6.638)\end{array}$ & $\begin{array}{c}23.651^{* *} \\
(10.691)\end{array}$ & $\begin{array}{c}6.355^{* * *} \\
(1.374)\end{array}$ & $\begin{array}{c}6.468^{* * *} \\
(1.417)\end{array}$ & $\begin{array}{c}4.855^{* * * *} \\
(1.865)\end{array}$ \\
\hline De jure exchange rate regime & $\begin{array}{l}7.220 \\
(5.844)\end{array}$ & $\begin{array}{c}26.917^{* * *} \\
(8.632)\end{array}$ & $\begin{array}{c}23.309^{* *} \\
(11.139)\end{array}$ & $\begin{array}{c}2.218 \\
(1.462)\end{array}$ & $\begin{array}{c}3.105^{* *} \\
(1.515)\end{array}$ & $\begin{array}{c}3.946^{* *} \\
(1.891)\end{array}$ \\
\hline De facto exchange rate regime & $\begin{array}{c}8.889 \\
(5.778)\end{array}$ & $\begin{array}{c}27.651^{* * *} \\
(8.363)\end{array}$ & $\begin{array}{c}23.645^{* *} \\
(10.742)\end{array}$ & $\begin{array}{l}2.784^{*} \\
(1.442)\end{array}$ & $\begin{array}{c}3.724^{* *} \\
(1.502)\end{array}$ & $\begin{array}{c}4.892^{* * *} \\
(1.870)\end{array}$ \\
\hline Bank cost-to-income ratio & & $\begin{array}{c}31.088^{* * *} \\
(6.588)\end{array}$ & $\begin{array}{c}24.737^{* *} \\
(10.729)\end{array}$ & & $\begin{array}{c}6.735^{* * * *} \\
(1.413)\end{array}$ & $\begin{array}{l}4.810^{* *} \\
(1.881)\end{array}$ \\
\hline Bank overhead costs & & $\begin{array}{c}32.479^{* * *} \\
(6.608)\end{array}$ & $\begin{array}{l}17.681^{*} \\
(10.667)\end{array}$ & & $\begin{array}{c}6.912^{* * *} \\
(1.422)\end{array}$ & $\begin{array}{l}4.625^{* *} \\
(1.896)\end{array}$ \\
\hline Income diversification & & $\begin{array}{c}31.614^{* * *} \\
(6.658)\end{array}$ & $\begin{array}{c}25.367^{* *} \\
(10.728)\end{array}$ & & $\begin{array}{c}6.850^{* * * *} \\
(1.435)\end{array}$ & $\begin{array}{c}5.138^{* * *} \\
(1.859)\end{array}$ \\
\hline Foreign bank presence & & $\begin{array}{c}26.670^{* * *} \\
(6.467)\end{array}$ & $\begin{array}{l}19.666^{*} \\
(10.803)\end{array}$ & & $\begin{array}{c}6.524^{* * *} \\
(1.426)\end{array}$ & $\begin{array}{l}4.777^{* *} \\
(1.900)\end{array}$ \\
\hline State-owned bank presence & & $\begin{array}{c}25.870^{* * *} \\
(4.997)\end{array}$ & $\begin{array}{c}20.825^{* * *} \\
(5.878)\end{array}$ & & $\begin{array}{c}7.244^{* * * *} \\
(1.409)\end{array}$ & $\begin{array}{c}6.291^{* * *} \\
(1.934)\end{array}$ \\
\hline De facto financial openness & & & $\begin{array}{c}22.978^{* *} \\
(10.800) \\
\end{array}$ & & & $\begin{array}{l}4.841^{* *} \\
(1.883)\end{array}$ \\
\hline Measure of central banks' preferences & \multicolumn{6}{|c|}{ CONS_W } \\
\hline Dependent variable & \multicolumn{3}{|c|}{ Credit-to-deposit ratio } & \multicolumn{3}{|c|}{ Nonperforming loans ratio } \\
\hline & (1) & $(2)$ & (3) & $(1)$ & $(2)$ & $(3)$ \\
\hline Additional control variable & & & & & & \\
\hline Central bank independence & $\begin{array}{c}13.005^{* *} \\
(5.561)\end{array}$ & $\begin{array}{c}25.637^{* * *} \\
(6.953)\end{array}$ & $\begin{array}{c}23.526^{* *} \\
(10.994)\end{array}$ & $\begin{array}{c}5.589^{* * *} \\
(1.265)\end{array}$ & $\begin{array}{c}5.619^{* * *} \\
(1.304)\end{array}$ & $\begin{array}{c}4.851^{* *} \\
(2.016)\end{array}$ \\
\hline Partial adoption of inflation targeting & $\begin{array}{c}14.084^{* * *} \\
(5.356)\end{array}$ & $\begin{array}{c}25.589^{* * *} \\
(6.713)\end{array}$ & $\begin{array}{c}25.529 * * \\
(10.554)\end{array}$ & $\begin{array}{c}6.173^{* * *} \\
(1.404)\end{array}$ & $\begin{array}{c}5.783^{* * * *} \\
(1.458)\end{array}$ & $\begin{array}{l}4.735^{* *} \\
(1.889)\end{array}$ \\
\hline Fully-fledged inflation targeting & $\begin{array}{c}14.216^{* * *} \\
(5.352)\end{array}$ & $\begin{array}{c}25.526^{* * * *} \\
(6.702)\end{array}$ & $\begin{array}{c}26.413^{* *} \\
(10.502)\end{array}$ & $\begin{array}{c}6.255^{* * *} \\
(1.402)\end{array}$ & $\begin{array}{c}5.873^{* * *} \\
(1.456)\end{array}$ & $\begin{array}{c}5.052^{* * *} \\
(1.881)\end{array}$ \\
\hline De jure exchange rate regime & $\begin{array}{c}5.003 \\
(5.709)\end{array}$ & $\begin{array}{c}26.172^{* * * *} \\
(8.568)\end{array}$ & $\begin{array}{c}25.730^{* *} \\
(10.882)\end{array}$ & $\begin{array}{l}2.539^{*} \\
(1.501)\end{array}$ & $\begin{array}{c}3.211^{* *} \\
(1.554)\end{array}$ & $\begin{array}{l}4.051^{* *} \\
(1.904)\end{array}$ \\
\hline De facto exchange rate regime & $\begin{array}{c}6.841 \\
(5.638)\end{array}$ & $\begin{array}{c}27.043^{* * *} \\
(8.347)\end{array}$ & $\begin{array}{c}25.946^{* *} \\
(10.536)\end{array}$ & $\begin{array}{c}3.096^{* *} \\
(1.482)\end{array}$ & $\begin{array}{c}3.856^{* *} \\
(1.542)\end{array}$ & $\begin{array}{c}4.955^{* * *} \\
(1.886)\end{array}$ \\
\hline Bank cost-to-income ratio & & $\begin{array}{c}25.393^{* * *} \\
(6.665)\end{array}$ & $\begin{array}{c}26.680^{* *} \\
(10.529)\end{array}$ & & $\begin{array}{c}6.075^{* * *} \\
(1.459)\end{array}$ & $\begin{array}{c}4.928^{* * *} \\
(1.894)\end{array}$ \\
\hline Bank overhead costs & & $\begin{array}{c}26.673^{* * *} \\
(6.690)\end{array}$ & $\begin{array}{l}19.742^{*} \\
(10.512)\end{array}$ & & $\begin{array}{c}6.228^{* * * *} \\
(1.471)\end{array}$ & $\begin{array}{c}4.699^{* *} \\
(1.912)\end{array}$ \\
\hline Income diversification & & $\begin{array}{c}25.568^{* * *} \\
(6.720)\end{array}$ & $\begin{array}{c}27.305^{* *} \\
(10.527)\end{array}$ & & $\begin{array}{c}6.005^{* * * *} \\
(1.469)\end{array}$ & $\begin{array}{c}5.123^{* * *} \\
(1.871)\end{array}$ \\
\hline Foreign bank presence & & $\begin{array}{c}22.193^{* * *} \\
(6.497)\end{array}$ & $\begin{array}{c}22.833^{* *} \\
(10.540)\end{array}$ & & $\begin{array}{c}5.806^{* * *} \\
(1.466)\end{array}$ & $\begin{array}{c}4.848^{* *} \\
(1.904)\end{array}$ \\
\hline State-owned bank presence & & $\begin{array}{c}19.211^{* * *} \\
(5.213)\end{array}$ & $\begin{array}{c}21.111^{* * *} \\
(5.951)\end{array}$ & & $\begin{array}{c}6.092^{* * *} \\
(1.470)\end{array}$ & $\begin{array}{c}6.066^{* * *} \\
(1.991)\end{array}$ \\
\hline De facto financial openness & & & $\begin{array}{c}24.852^{* *} \\
(10.657)\end{array}$ & & & $\begin{array}{c}5.006^{* * *} \\
(1.905)\end{array}$ \\
\hline
\end{tabular}

Note: Standard errors are reported in parentheses. *; **, and *** indicate statistical significance at the $10 \%, 5 \%$ and $1 \%$ level, respectively. 
Table 7: Robustness checks - Additional control variables: Results obtained with the Z-score and the capital-to-asset ratio

\begin{tabular}{|c|c|c|c|c|c|c|}
\hline \multirow{3}{*}{$\begin{array}{l}\text { Measure of central banks' preferences } \\
\text { Dependent variable }\end{array}$} & \multicolumn{6}{|c|}{ CONS } \\
\hline & \multicolumn{3}{|c|}{ Z-score } & \multicolumn{3}{|c|}{ Capital-to-asset ratio } \\
\hline & (1) & (2) & (3) & (1) & (2) & (3) \\
\hline \multicolumn{7}{|l|}{ Additional control variable } \\
\hline Central bank independence & $\begin{array}{c}-2.286^{* *} \\
(1.160)\end{array}$ & $\begin{array}{c}-2.231^{*} \\
(1.266)\end{array}$ & $\begin{array}{c}-4.274^{*} \\
(2.366)\end{array}$ & $\begin{array}{c}-2.973^{* * *} \\
(0.626)\end{array}$ & $\begin{array}{c}-2.048^{* * *} \\
(0.607)\end{array}$ & $\begin{array}{l}-1.227 \\
(1.055)\end{array}$ \\
\hline Partial adoption of inflation targeting & $\begin{array}{c}-2.541^{* *} \\
(1.104)\end{array}$ & $\begin{array}{c}-2.625^{* *} \\
(1.189)\end{array}$ & $\begin{array}{c}-5.159^{* *} \\
(2.288)\end{array}$ & $\begin{array}{l}-2.902^{* * *} \\
(0.599)\end{array}$ & $\begin{array}{c}-1.977^{* * *} \\
(0.581)\end{array}$ & $\begin{array}{l}-0.948 \\
(1.055)\end{array}$ \\
\hline Fully-fledged inflation targeting & $\begin{array}{c}-2.495^{* *} \\
(1.100)\end{array}$ & $\begin{array}{c}-2.586^{* *} \\
(1.185)\end{array}$ & $\begin{array}{c}-4.540^{* *} \\
(2.275)\end{array}$ & $\begin{array}{c}-3.000 * * * \\
(0.601)\end{array}$ & $\begin{array}{c}-2.104^{* * *} \\
(0.584)\end{array}$ & $\begin{array}{l}-1.231 \\
(1.061)\end{array}$ \\
\hline De jure exchange rate regime & $\begin{array}{c}-2.655 \\
(1.637)\end{array}$ & $\begin{array}{c}-2.561 \\
(1.783)\end{array}$ & $\begin{array}{c}-4.930^{* *} \\
(2.384)\end{array}$ & $\begin{array}{c}-2.224^{* * *} \\
(0.841)\end{array}$ & $\begin{array}{c}-1.933^{* *} \\
(0.821)\end{array}$ & $\begin{array}{l}-1.781 \\
(1.081)\end{array}$ \\
\hline De facto exchange rate regime & $\begin{array}{l}-2.355 \\
(1.593)\end{array}$ & $\begin{array}{l}-2.267 \\
(1.737)\end{array}$ & $\begin{array}{c}-4.429^{*} \\
(2.308)\end{array}$ & $\begin{array}{c}-1.889^{* *} \\
(0.835)\end{array}$ & $\begin{array}{c}-1.550^{*} \\
(0.822)\end{array}$ & $\begin{array}{l}-1.171 \\
(1.051)\end{array}$ \\
\hline Bank cost-to-income ratio & & $\begin{array}{c}-2.157^{*} \\
(1.192)\end{array}$ & $\begin{array}{c}-4.054^{*} \\
(2.300)\end{array}$ & & $\begin{array}{c}-1.948^{* * *} \\
(0.583)\end{array}$ & $\begin{array}{l}-1.152 \\
(1.054)\end{array}$ \\
\hline Bank overhead costs & & $\begin{array}{l}-1.991^{*} \\
(1.200)\end{array}$ & $\begin{array}{c}-4.983^{* *} \\
(2.324)\end{array}$ & & $\begin{array}{c}-2.049 * * * \\
(0.584)\end{array}$ & $\begin{array}{l}-0.743 \\
(1.095)\end{array}$ \\
\hline Income diversification & & $\begin{array}{c}-2.425^{* *} \\
(1.206)\end{array}$ & $\begin{array}{c}-4.735^{* *} \\
(2.297)\end{array}$ & & $\begin{array}{c}-1.930^{* * *} \\
(0.593)\end{array}$ & $\begin{array}{l}-1.087 \\
(1.058)\end{array}$ \\
\hline Foreign bank presence & & $\begin{array}{c}-2.376^{* *} \\
(1.201)\end{array}$ & $\begin{array}{c}-4.875^{* *} \\
(2.325)\end{array}$ & & $\begin{array}{c}-1.926^{* * *} \\
(0.587)\end{array}$ & $\begin{array}{l}-0.449 \\
(1.086)\end{array}$ \\
\hline State-owned bank presence & & $\begin{array}{c}-2.075^{* *} \\
(1.000)\end{array}$ & $\begin{array}{c}-1.996 \\
(1.985)\end{array}$ & & $\begin{array}{c}-1.581^{* *} \\
(0.645)\end{array}$ & $\begin{array}{c}0.938 \\
(1.174)\end{array}$ \\
\hline De facto financial openness & & & $\begin{array}{c}-4.319^{*} \\
(2.313)\end{array}$ & & & $\begin{array}{l}-1.059 \\
(1.054)\end{array}$ \\
\hline Measure of central banks' preferences & \multicolumn{6}{|c|}{ CONS_W } \\
\hline \multirow[t]{2}{*}{ Dependent variable } & \multicolumn{3}{|c|}{ Z Z-score } & \multicolumn{3}{|c|}{ Capital-to-asset ratio } \\
\hline & (1) & $(2)$ & $(3)$ & $(1)$ & $(2)$ & $(3)$ \\
\hline \\
\hline $\begin{array}{l}\text { Additional control variable } \\
\text { Central bank independence }\end{array}$ & $\begin{array}{c}-3.309^{* * *} \\
(1.143)\end{array}$ & $\begin{array}{c}-3.625^{* * *} \\
(1.284)\end{array}$ & $\begin{array}{c}-4.677^{* *} \\
(2.348)\end{array}$ & $\begin{array}{c}-3.065^{* * *} \\
(0.647)\end{array}$ & $\begin{array}{c}-2.016^{* * *} \\
(0.640)\end{array}$ & $\begin{array}{l}-1.037 \\
(1.168)\end{array}$ \\
\hline Partial adoption of inflation targeting & $\begin{array}{c}-3.401^{* * *} \\
(1.078)\end{array}$ & $\begin{array}{c}-3.775^{* * *} \\
(1.193)\end{array}$ & $\begin{array}{c}-5.277^{* *} \\
(2.252)\end{array}$ & $\begin{array}{c}-3.070^{* * *} \\
(0.615)\end{array}$ & $\begin{array}{c}-2.003^{* * *} \\
(0.608)\end{array}$ & $\begin{array}{l}-0.789 \\
(1.163)\end{array}$ \\
\hline Fully-fledged inflation targeting & $\begin{array}{c}-3.361^{* * *} \\
(1.075)\end{array}$ & $\begin{array}{c}-3.656^{* * *} \\
(1.189)\end{array}$ & $\begin{array}{c}-4.553^{* *} \\
(2.244)\end{array}$ & $\begin{array}{l}-3.135^{* * *} \\
(0.616)\end{array}$ & $\begin{array}{c}-2.064^{* * *} \\
(0.609)\end{array}$ & $\begin{array}{l}-1.070 \\
(1.161)\end{array}$ \\
\hline De jure exchange rate regime & $\begin{array}{l}-3.006^{*} \\
(1.580)\end{array}$ & $\begin{array}{c}-3.231^{*} \\
(1.774)\end{array}$ & $\begin{array}{c}-5.125^{* *} \\
(2.338)\end{array}$ & $\begin{array}{c}-2.464^{* * *} \\
(0.907)\end{array}$ & $\begin{array}{c}-1.818^{* *} \\
(0.897)\end{array}$ & $\begin{array}{l}-1.673 \\
(1.187)\end{array}$ \\
\hline De facto exchange rate regime & $\begin{array}{l}-2.724^{*} \\
(1.545)\end{array}$ & $\begin{array}{c}-2.945^{*} \\
(1.736)\end{array}$ & $\begin{array}{c}-4.665^{* *} \\
(2.272)\end{array}$ & $\begin{array}{c}-2.122^{* *} \\
(0.904)\end{array}$ & $\begin{array}{l}-1.418 \\
(0.900)\end{array}$ & $\begin{array}{l}-1.065 \\
(1.157)\end{array}$ \\
\hline Bank cost-to-income ratio & & $\begin{array}{c}-3.464^{* * *} \\
(1.199)\end{array}$ & $\begin{array}{c}-4.408^{*} \\
(2.264)\end{array}$ & & $\begin{array}{c}-1.966^{* * *} \\
(0.611)\end{array}$ & $\begin{array}{l}-1.027 \\
(1.160)\end{array}$ \\
\hline Bank overhead costs & & $\begin{array}{c}-3.300^{* * *} \\
(1.209)\end{array}$ & $\begin{array}{c}-5.269^{* *} \\
(2.294)\end{array}$ & & $\begin{array}{c}-2.079^{* * *} \\
(0.613)\end{array}$ & $\begin{array}{l}-0.483 \\
(1.226)\end{array}$ \\
\hline Income diversification & & $\begin{array}{c}-3.609 * * * \\
(1.206)\end{array}$ & $\begin{array}{c}-4.962^{* *} \\
(2.259)\end{array}$ & & $\begin{array}{c}-1.948^{* * *} \\
(0.614)\end{array}$ & $\begin{array}{l}-0.987 \\
(1.161)\end{array}$ \\
\hline Foreign bank presence & & $\begin{array}{c}-3.588^{* * *} \\
(1.205)\end{array}$ & $\begin{array}{c}-4.985^{* *} \\
(2.280)\end{array}$ & & $\begin{array}{c}-1.948^{* * *} \\
(0.613)\end{array}$ & $\begin{array}{l}-0.323 \\
(1.183)\end{array}$ \\
\hline State-owned bank presence & & $\begin{array}{c}-2.824^{* * *} \\
(1.022)\end{array}$ & $\begin{array}{l}-0.825 \\
(2.038)\end{array}$ & & $\begin{array}{c}-1.805^{* * *} \\
(0.673)\end{array}$ & $\begin{array}{c}0.728 \\
(1.294)\end{array}$ \\
\hline De facto financial openness & & & $\begin{array}{c}-4.701^{* *} \\
(2.287)\end{array}$ & & & $\begin{array}{l}-0.962 \\
(1.163)\end{array}$ \\
\hline
\end{tabular}

Note: Standard errors are reported in parentheses. $*$; **, and $* * *$ indicate statistical significance at the $10 \%, 5 \%$ and $1 \%$ level, respectively. 
Alternative measure of central banks' preferences. We develop an alternative measure of central banks' preferences which control for the fact that the monetary policy dependence vis-à-vis the rest of the world and the government's preferences could impact the output gap and inflation volatility, and then the CONS and CONS_W indexes. From a broader perspective, the monetary policy dependence vis-à-vis the rest of the world relies to the well-known monetary policy "trilemma" (Mundell, 1963), according to which a country cannot have simultaneously free capital mobility, exchange rate management and monetary autonomy. Countries may choose only two of the three policy goals. Consequently, such a framework implies that the behavior of the central bank is constrained by at least one side of the "impossible trinity". For instance, in the most extreme case, the literature on the open-economy "trilemma" suggests that a country facing a completely open capital account and a fixed exchange rate regime must align its interest rate policy to exactly match that of its base country (Frankel et al., 2004). This means that the output gap and inflation volatility in small open economies could be partly explained by interest rate fluctuations in large influential economies, i.e. countries to which currencies are typically pegged.

Consequently, we compute two alternative measures of central banks' preferences, respectively called "cleansed CONS" and "cleansed CONS_W", in order to consider these potential interferences. Indeed, these two measures can be viewed as the part of the original indexes that cannot be explained by the trilemma configuration and the government's preferences. The details concerning the methodology used to compute these alternative measures of central banks' preferences are provided in Appendix 3. Results obtained with the "cleansed CONS" (or "cleansed CONS_W") as right-hand side variable are reported in table 8 . They are similar to those obtained with the original measures of central banks' preferences.

Table 8: Robustness checks - Alternative measures of central banks' preferences: Results obtained with "cleansed" CONS and "cleansed" CONS_W

\begin{tabular}{l|ccc|ccc}
\hline \hline Dependent variable & \multicolumn{3}{|c|}{ Credit volatility } & \multicolumn{3}{c}{ Credit-to-GDP gap } \\
& $(1)$ & $(2)$ & $(3)$ & $(1)$ & $(2)$ & $(3)$ \\
\hline Cleansed CONS & $46.648^{* *}$ & $124.362^{* * *}$ & $87.187^{* * *}$ & $25.341^{* * *}$ & $30.040^{* * *}$ & $25.476^{* *}$ \\
& $(23.220)$ & $(40.592)$ & $(26.830)$ & $(5.715)$ & $(7.234)$ & $(11.358)$ \\
Cleansed CONS_W & $57.189^{* *}$ & $130.622^{* * *}$ & $86.455^{* * *}$ & $19.893^{* * *}$ & $26.762^{* * *}$ & $27.327^{* *}$ \\
& $(22.682)$ & $(40.761)$ & $(26.357)$ & $(5.609)$ & $(7.237)$ & $(11.129)$ \\
\hline & \multicolumn{3}{|c|}{ Credit-to-deposit ratio } & Nonperforming loans ratio \\
& $(1)$ & $(2)$ & $(3)$ & $(1)$ & $(2)$ & $(3)$ \\
\hline Cleansed CONS & $36.161^{* * *}$ & $62.040^{* * *}$ & $43.936^{* *}$ & $12.214^{* * *}$ & $12.356^{* * *}$ & $9.628^{* *}$ \\
& $(11.439)$ & $(13.390)$ & $(21.555)$ & $(2.847)$ & $(2.909)$ & $(3.769)$ \\
Cleansed CONS_W & $25.177^{* *}$ & $49.631^{* * *}$ & $48.005^{* *}$ & $11.606^{* * *}$ & $10.711^{* * *}$ & $9.830^{* * *}$ \\
& $(11.202)$ & $(13.392)$ & $(21.059)$ & $(2.872)$ & $(2.956)$ & $(3.780)$ \\
\hline & \multicolumn{3}{c}{ Z-score } & & \multicolumn{3}{c}{ Capital-to-asset ratio } \\
& $(1)$ & $(2)$ & $(3)$ & $(1)$ & $(2)$ & $(3)$ \\
\hline Cleansed CONS & $-4.147^{*}$ & -3.552 & -7.392 & $-5.051^{* * *}$ & $-3.860^{* * *}$ & -1.882 \\
& $(2.246)$ & $(2.436)$ & $(4.669)$ & $(1.258)$ & $(1.197)$ & $(2.150)$ \\
Cleansed CONS_W & $-6.199^{* * *}$ & $-6.312^{* * *}$ & $-8.100^{*}$ & $-5.386^{* * *}$ & $-3.907^{* * *}$ & -1.493 \\
& $(2.178)$ & $(2.422)$ & $(4.577)$ & $(1.270)$ & $(1.226)$ & $(2.320)$ \\
\hline \hline
\end{tabular}

Note: Standard errors are reported in parentheses. *; **, and *** indicate statistical significance at the $10 \%, 5 \%$ and $1 \%$ level, respectively. 
Endogeneity issue. Finally, it can be argued that there might be a potential reverse causality from banking sector vulnerability to the preferences of central banks. To address this potential endogeneity issue, we further consider an instrumental variable approach using the two-stage least squares (2SLS) estimator. Three instrumental variables are considered: the first lag of the CONS (or CONS_W) index, and two complementary proxies for central bank independence, namely the CWN index and the turnover rate of central bank governors.

Instrumental variables estimates for each measure of banking sector vulnerability and each specification are reported in Tables 9 and 10. As above, to save space we only report the coefficients for CONS and CONS_W. As we can see, the results after correcting for potential endogeneity are very similar to our previous findings as we still find a significant relationship between the preferences of central banks and banking sector vulnerability. The effect of the preferences of central banks appears to be even stronger than with the fixed-effects estimator. Note that the Hansen test p-values and the Cragg-Donald statistics indicate that our instruments are valid and not weak ${ }^{25}$.

Overall, these additional results reinforce the finding that a high degree of central bank conservatism exacerbates the vulnerability of the banking sector, which is in line with the benign neglect hypothesis.

\footnotetext{
${ }^{25}$ Please note that we obtain similar results when we consider the "cleansed" CONS and CONS_W indexes as measures of central banks' preferences, with the first lag of the "cleansed" CONS (or CONS_W) index, the CWN index and the turnover rate of central bank governors as instrumental variables. Results are available upon request.
} 
Table 9: 2SLS results for credit volatility, credit-to-GDP gap and credit-to-deposit ratio

\begin{tabular}{|c|c|c|c|c|c|c|}
\hline \multirow[t]{2}{*}{ Dependent variable } & \multicolumn{6}{|c|}{ Credit volatility } \\
\hline & (1) & $(2)$ & (3) & (1) & $(2)$ & $(3)$ \\
\hline CONS & $\begin{array}{c}40.181^{* * * *} \\
(13.473)\end{array}$ & $\begin{array}{c}110.949^{* * *} \\
(26.289)\end{array}$ & $\begin{array}{c}55.870^{* * *} \\
(16.824)\end{array}$ & & & \\
\hline CONS_W & & & & $\begin{array}{c}49.668^{* * *} \\
(14.777)\end{array}$ & $\begin{array}{c}123.588^{* * *} \\
(29.544)\end{array}$ & $\begin{array}{c}57.499^{* * *} \\
(17.444)\end{array}$ \\
\hline Observations & 835 & 485 & 321 & 769 & 454 & 298 \\
\hline Number of countries & 67 & 56 & 46 & 65 & 54 & 44 \\
\hline R-squared & 0.046 & 0.068 & 0.117 & 0.048 & 0.061 & 0.112 \\
\hline Hansen J-OverID test [p-value] & 0.729 & 0.336 & 0.0981 & 0.884 & 0.399 & 0.160 \\
\hline Cragg-Donald Wald F Stat. & 563.4 & 242.8 & 126.4 & 342.2 & 161.9 & 105.3 \\
\hline \multirow[t]{3}{*}{ Stock \& Yogo critical value $(10 \%)$} & 22.30 & 22.30 & 22.30 & 22.30 & 22.30 & 22.30 \\
\hline & \multicolumn{6}{|c|}{ Credit-to-GDP gap } \\
\hline & (1) & $(2)$ & (3) & (1) & $(2)$ & $(3)$ \\
\hline CONS & $\begin{array}{c}13.312^{* * *} \\
(3.197)\end{array}$ & $\begin{array}{c}16.256^{* * *} \\
(4.267)\end{array}$ & $\begin{array}{c}14.307^{* *} \\
(7.066)\end{array}$ & & & \\
\hline CONS_W & & & & $\begin{array}{c}9.328^{* * *} \\
(3.620)\end{array}$ & $\begin{array}{c}11.727^{* *} \\
(4.784)\end{array}$ & $\begin{array}{c}14.986^{* *} \\
(7.631)\end{array}$ \\
\hline Observations & 946 & 588 & 329 & 881 & 558 & 306 \\
\hline Number of countries & 68 & 61 & 50 & 67 & 60 & 48 \\
\hline R-squared & 0.143 & 0.228 & 0.280 & 0.119 & 0.214 & 0.268 \\
\hline Hansen J-OverID test [p-value] & 0.208 & 0.590 & 0.0649 & 0.208 & 0.469 & 0.0742 \\
\hline Cragg-Donald Wald F Stat. & 730.3 & 364.1 & 128.3 & 441.5 & 243.4 & 106.5 \\
\hline \multirow[t]{3}{*}{ Stock \& Yogo critical value $(10 \%)$} & 22.30 & 22.30 & 22.30 & 22.30 & 22.30 & 22.30 \\
\hline & \multicolumn{6}{|c|}{ Credit-to-deposit ratio } \\
\hline & (1) & $(2)$ & $(3)$ & $(1)$ & $(2)$ & $(3)$ \\
\hline CONS & $\begin{array}{c}16.175^{* *} \\
(6.549)\end{array}$ & $\begin{array}{c}34.659^{* * *} \\
(8.104)\end{array}$ & $\begin{array}{c}28.435^{* *} \\
(13.471)\end{array}$ & & & \\
\hline CONS_W & & & & $\begin{array}{l}10.367 \\
(7.377)\end{array}$ & $\begin{array}{c}24.469^{* * *} \\
(9.297)\end{array}$ & $\begin{array}{c}29.426^{* *} \\
(14.961)\end{array}$ \\
\hline Observations & 892 & 540 & 307 & 828 & 510 & 284 \\
\hline Number of countries & 67 & 56 & 45 & 66 & 55 & 43 \\
\hline R-squared & 0.157 & 0.215 & 0.241 & 0.148 & 0.185 & 0.227 \\
\hline Hansen J-OverID test [p-value] & 0.0497 & 0.474 & 0.822 & 0.0546 & 0.744 & 0.745 \\
\hline Cragg-Donald Wald F Stat. & 648.2 & 306 & 125.2 & 391 & 199.3 & 100.3 \\
\hline Stock \& Yogo critical value $(10 \%)$ & 22.30 & 22.30 & 22.30 & 22.30 & 22.30 & 22.30 \\
\hline
\end{tabular}

Note: Standard errors are reported in parentheses. *; **, and ${ }^{* * *}$ indicate statistical significance at the $10 \%, 5 \%$ and $1 \%$ level, respectively. 
Table 10: 2SLS results for nonperforming loans ratio, Z-score and capital-to-asset ratio

\begin{tabular}{|c|c|c|c|c|c|c|}
\hline \multirow[t]{2}{*}{ Dependent variable } & \multicolumn{6}{|c|}{ Nonperforming loans ratio } \\
\hline & (1) & $(2)$ & $(3)$ & (1) & $(2)$ & $(3)$ \\
\hline CONS & $\begin{array}{c}10.459^{* * *} \\
(1.500)\end{array}$ & $\begin{array}{c}11.015^{\text {*** }} \\
(1.531)\end{array}$ & $\begin{array}{c}12.108^{* * * *} \\
(2.500)\end{array}$ & & & \\
\hline CONS_W & & & & $\begin{array}{c}9.636^{* * *} \\
(1.713)\end{array}$ & $\begin{array}{c}9.837^{* * *} \\
(1.758)\end{array}$ & $\begin{array}{c}10.075^{* * *} \\
(2.701)\end{array}$ \\
\hline Observations & 558 & 526 & 298 & 532 & 502 & 281 \\
\hline Number of countries & 54 & 52 & 42 & 54 & 52 & 42 \\
\hline R-squared & 0.366 & 0.396 & 0.355 & 0.360 & 0.380 & 0.353 \\
\hline Hansen J-OverID test [p-value] & 0.0469 & 0.0375 & 0.104 & 0.108 & 0.105 & 0.273 \\
\hline Cragg-Donald Wald F Stat. & 318.1 & 289.4 & 109.9 & 189.1 & 179.9 & 83.79 \\
\hline \multirow[t]{3}{*}{ Stock \& Yogo critical value $(10 \%)$} & 22.30 & 22.30 & 22.30 & 22.30 & 22.30 & 22.30 \\
\hline & \multicolumn{6}{|c|}{ Z-score } \\
\hline & $(1)$ & $(2)$ & $(3)$ & $(1)$ & $(2)$ & $(3)$ \\
\hline CONS & $\begin{array}{l}-2.398^{*} \\
(1.381)\end{array}$ & $\begin{array}{c}-2.301 \\
(1.489)\end{array}$ & $\begin{array}{l}-4.708 \\
(2.901)\end{array}$ & & & \\
\hline CONS_W & & & & $\begin{array}{c}-3.070^{* *} \\
(1.545)\end{array}$ & $\begin{array}{c}-3.243^{*} \\
(1.684)\end{array}$ & $\begin{array}{l}-3.252 \\
(3.192)\end{array}$ \\
\hline Observations & 692 & 597 & 329 & 656 & 567 & 306 \\
\hline Number of countries & 65 & 61 & 50 & 64 & 60 & 48 \\
\hline R-squared & 0.039 & 0.056 & 0.060 & 0.055 & 0.069 & 0.067 \\
\hline Hansen J-OverID test [p-value] & 0.207 & 0.475 & 0.637 & 0.192 & 0.470 & 0.583 \\
\hline Cragg-Donald Wald F Stat. & 430.4 & 371.9 & 128.3 & 274.1 & 247.7 & 106.5 \\
\hline \multirow[t]{3}{*}{ Stock \& Yogo critical value (10\%) } & 22.30 & 22.30 & 22.30 & 22.30 & 22.30 & 22.30 \\
\hline & \multicolumn{6}{|c|}{ Capital-to-asset ratio } \\
\hline & (1) & $(2)$ & $(3)$ & (1) & $(2)$ & $(3)$ \\
\hline CONS & $\begin{array}{c}-2.258^{* * *} \\
(0.745)\end{array}$ & $\begin{array}{l}-1.302^{*} \\
(0.714)\end{array}$ & $\begin{array}{l}-0.768 \\
(1.227)\end{array}$ & & & \\
\hline CONS_W & & & & $\begin{array}{c}-1.723^{* *} \\
(0.830)\end{array}$ & $\begin{array}{l}-0.947 \\
(0.795)\end{array}$ & $\begin{array}{c}-0.784 \\
(1.425)\end{array}$ \\
\hline Observations & 421 & 394 & 178 & 402 & 376 & 168 \\
\hline Number of countries & 50 & 48 & 36 & 50 & 48 & 36 \\
\hline R-squared & 0.126 & 0.177 & 0.203 & 0.121 & 0.177 & 0.263 \\
\hline Hansen J-OverID test [p-value] & 0.283 & 0.584 & 0.396 & 0.878 & 0.624 & 0.138 \\
\hline Cragg-Donald Wald F Stat. & 256.4 & 239.5 & 63.51 & 169.1 & 164.6 & 48.99 \\
\hline Stock \& Yogo critical value $(10 \%)$ & 22.30 & 22.30 & 22.30 & 22.30 & 22.30 & 22.30 \\
\hline
\end{tabular}

Note: Standard errors are reported in parentheses. ${ }^{*} ; * *$, and $* * *$ indicate statistical significance at the $10 \%, 5 \%$ and $1 \%$ level, respectively. 


\section{Conclusion}

The global financial crisis occurred in the context of the Great Moderation. This has shed doubt on the Schwartz's conventional wisdom according to which price stability should guarantee macroeconomic and financial stability. An alternative growing view contends that with monetary policies focused primarily on price stability, financial risks were left largely unaddressed. The belief in the "divine coincidence" has, in retrospect, been revealed to be benign neglect. As a consequence, financial instability has undermined macroeconomic stability despite inflation being low and stable. Nonetheless, a few recent papers consider that there is no trade-off between monetary and financial stability, and support the Schwartz's hypothesis.

In this context, our paper is the first to address directly the link between the relative preferences of central banks for the inflation stabilization objective and banking sector vulnerability. This leads us to test the Schwartz's hypothesis against the benign neglect hypothesis. Our results, from a sample of 73 industrialized and emerging countries, indicate that differences in central banks' conservatism (CBC) robustly explain crosscountry differences in banking sector vulnerability and unambiguously validate the benign neglect hypothesis.

On normative grounds, this result suggests two alternative perspectives for recommendations. One is that central bankers now know that it could be very costly to neglect financial and banking vulnerabilities. In particular, once a dramatic crisis occurs, the usual monetary policy orthodoxy must be renounced in favour of unconventional measures. This should instead preemptively lead central bankers to tolerate a dilution of their primary price stability objective in order to devote greater attention to output and financial stability. Ideally, this could be stated in law. Central banks would then officially be responsible for this goal.

The other perspective ${ }^{26}$ is that if single mandates remain the rule, the implementation of an efficient macroprudential policy framework may reduce the adverse effects of high CBC. Some efforts have been made in this direction since 2008. However, such a framework is certainly not a panacea in itself because it may interfere with monetary policy. Indeed, monetary and macroprudential policies can be complementary, but they can also compete with one another, so they need to be coordinated. While the literature on this topic remains scarce, it is clear that the terms of the optimal coordination will depend on the preferences of the single or various authorities responsible for the two goals. In particular, the degree of conservatism of the central bank would influence the terms of the coordination and the corresponding macroeconomic equilibrium. In this respect, our results call for an analysis of the occurrence of trade-offs, with reference to the preferences of the authorities, given different types of shocks and given the underlying structural features of the economies.

While a higher level of CBC implies a more vulnerable banking sector, it is widely recognized that a highly inflationary context is not conducive to sound financial conditions. This suggests that an immediate extension of our results would be to examine the existence of non-linearities in the link between CBC and banking sector vulnerability. Furthermore, our results suggest more fundamental extensions. While this paper documents the ex ante effect of $\mathrm{CBC}$ (i.e. on financial vulnerabilities), it can be expected that the degree of $\mathrm{CBC}$ also impacts the pace of economic recovery in the aftermath of a crisis. Indeed, a conservative central banker may be reluctant to

\footnotetext{
${ }^{26}$ See for instance Svensson (2012) and Woodford (2012) for two different viewpoints on this issue.
} 
deviate from the sacred inflation objective to support the economy and the financial system once a financial crisis has occurred. At best, conservative monetary authorities would react too late ${ }^{27}$.

It is all the more important to assess whether CBC matters for the costs of crises, as the inflation targeting (IT) strategy has become very popular. While such a strategy can be followed in a flexible way, it firmly places the inflation objective at the heart of the monetary policy arrangements (Levieuge and Lucotte, 2013; King, 1997). Thus far, there is some debate on the performance of IT with respect to financial instability and the costs of crises. One reason may be that beyond the focus on inflation, the IT strategy is accompanied by institutional, political, legal and practical reforms that are globally beneficial to macroeconomic and financial stability. In emerging countries in particular, these reforms could overcome the negative effect of greater conservatism, at least in the first years following the adoption of IT. This is less obvious for industrialized countries, in which the aversion of central banks to inflation is already high and inflation has been under control for almost 30 years. While it is difficult to control for the effects of institutional improvement, it would be interesting to re-examine the empirical literature on the performance of IT by considering the relationship between IT, CBC and financial instability separately for developed and emerging countries.

${ }^{27}$ Such a view is supported, for example, by Whelan (2013, pp.107-108). 


\section{Appendix}

\section{Appendix 1 - Details on the CONS index}

Our measure of CBC uses the method suggested by Levieuge and Lucotte (2014) on the theoretical basis of the Taylor curve (Taylor, 1979). This curve, shown in Figure A1 below, represents the standard trade-off between the variability of the inflation rate $\left(\sigma_{\pi}^{2}\right)$ and the variability of the output gap $\left(\sigma_{y}^{2}\right)$. Theoretically, any point on this curve is the result of an optimal monetary policy, given the structural model of the economy and the weight assigned to the objective of inflation stabilization. Then, the position where an economy is observed on this curve reveals the central bank's preferences for inflation stabilization relative to output stabilization. The $45^{\circ}$ line corresponds to the case in which monetary authorities assign an equal weight to inflation and output variability in their loss function. A central bank is then considered increasingly conservative as its corresponding point moves along the Taylor curve from the right to the left. It suggests that inflation receives increasingly greater weight relative to output variability in its loss function. For example, point A in Figure A1 illustrates the case in which the central bank is more averse to inflation variability than at point B, while tolerating higher output variability. Point A then indicates a more conservative stance than point $\mathrm{B}$.

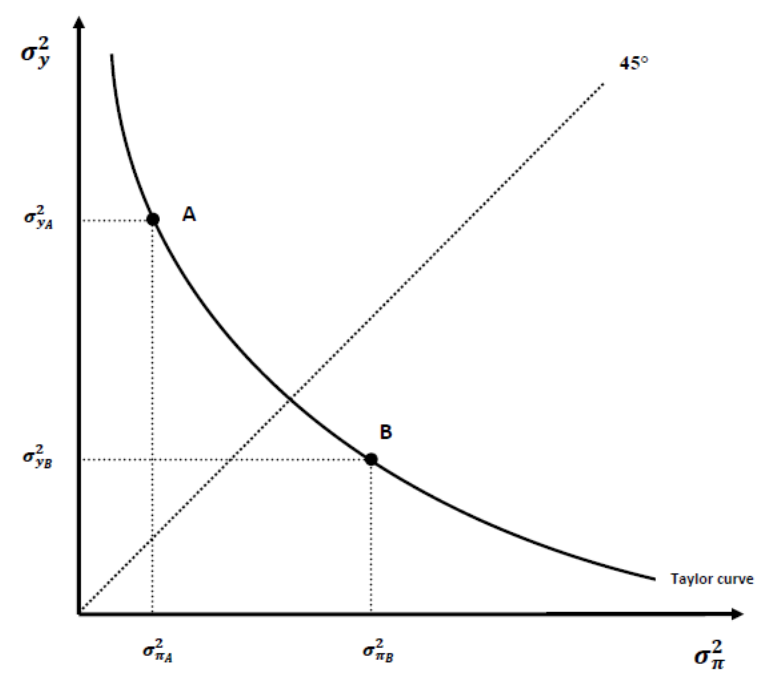

Figure A1: Preferences along the Taylor Curve

Following this conceptual background, Levieuge and Lucotte (2014) propose a new index, called $C O N S$, which is based on the value of the angle of the straight line joining the origin and a given point on the Taylor curve. Indeed, knowing the empirical volatilities of inflation and output gap on the adjacent and opposite sides respectively, it is possible to calculate the value of any angle using standard trigonometric formula: $\operatorname{angle}(\alpha)=\operatorname{atan}\left(\sigma_{y}^{2} / \sigma_{\pi}^{2}\right) \times 180 / p i$. Once rescaled to $[0,1]$, this angle measure constitutes a fair estimate of the relative degree of $\mathrm{CBC}$, equivalent to the relative weight assigned to the inflation objective in a standard quadratic loss function. Thus, CONS is defined as 


$$
C O N S=\frac{1}{90}\left[\operatorname{atan}\left(\frac{\sigma_{y}^{2}}{\sigma_{\pi}^{2}}\right) \times \frac{180}{p i}\right]
$$

Levieuge and Lucotte (2014) initially developed such a CONS index for the OECD countries. As $\left(\sigma_{\pi}^{2}\right)$ and $\left(\sigma_{y}^{2}\right)$ are easily observable in any country, over any period, extending this index to a broad set of countries is direct and simple. For the purposes of this paper, we have expanded the CONS index to a large set of 73 countries from 1980 to 2012. CONS is computed on an annual basis, with $\sigma_{\pi}^{2}$ and $\sigma_{y}^{2}$ computed over five-year rolling windows. As highlighted by Levieuge and Lucotte (2014), any change in $C O N S$ can be the result of disturbances, outside the willingness of the central bank to change its preferences. This is potentially an important point to address, as our sample includes emerging countries that are known to be subject to shocks. In this respect, Levieuge and Lucotte (2014) propose an alternative CBC indicator, labelled CONS_W (" $W$ " for weighted), where the ratio $\sigma_{y}^{2} / \sigma_{\pi}^{2}$ in Equation (2) is weighted by the ratio of disturbances $\sigma_{\varepsilon y}^{2} / \sigma_{\varepsilon \pi}^{2} . \quad \sigma_{\varepsilon y}^{2}$ and $\sigma_{\varepsilon \pi}^{2}$ are the variance of demand and supply shocks, respectively. They are identified from bivariate structural VAR models through the reliable decomposition scheme suggested by Blanchard and Quah (1989). Details are provided in Levieuge and Lucotte (2014).

While prudence requires a priori that cyclical shocks be taken into account, Figure A3 below shows that the two measures are highly correlated at least in their mean values.
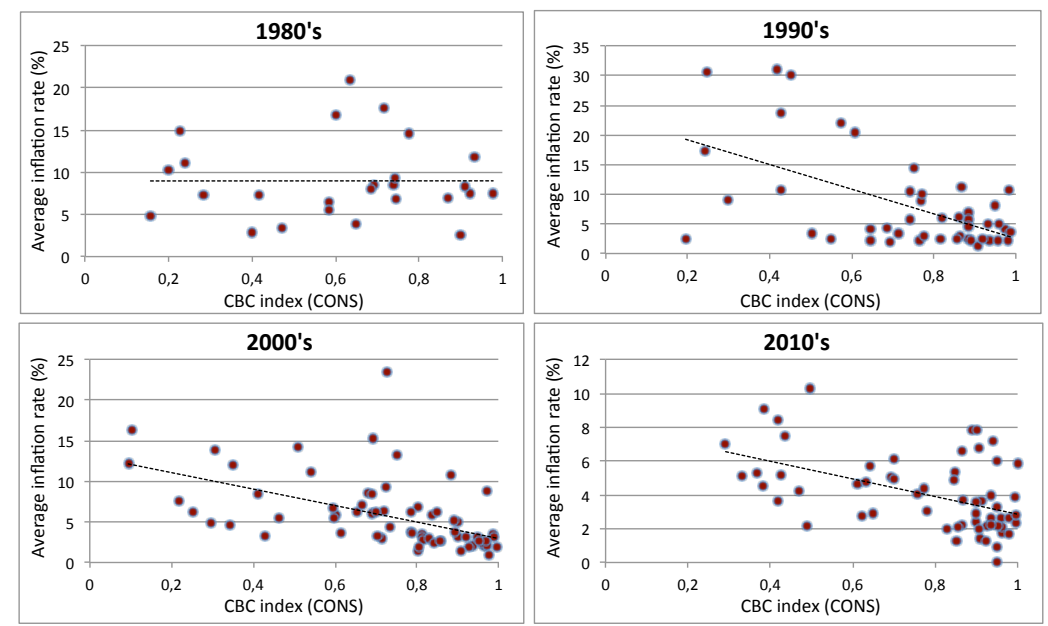

Figure A2: CONS index and inflation (decade average)

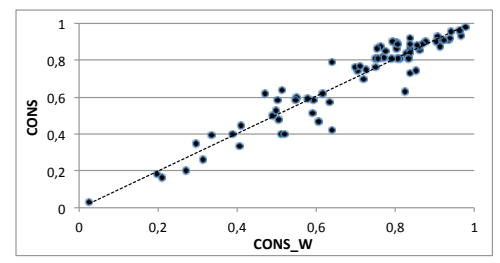

Figure A3: Correlation between $C O N S$ and $C O N S \_W$ (decade average) 


\section{Appendix 2 - Countries and average $C O N S$ and $C O N S \_W$}

Table A1: Average CONS and CONS_W

\begin{tabular}{|c|c|c|c|c|c|c|}
\hline Decade & \multicolumn{2}{|c|}{ 1980's } & \multicolumn{2}{|c|}{ 1990's } & \multicolumn{2}{|c|}{ 2000's } \\
\hline Country Name & CONS & $C O N S \_W$ & $C O N S$ & CONS_W & $C O N S$ & CONS_W \\
\hline Algeria & & & & & 0.405 & 0.335 \\
\hline Argentina & & & 0.978 & & 0.711 & 0.765 \\
\hline Armenia & & & & & 0.836 & 0.920 \\
\hline Australia & 0.740 & 0.823 & 0.816 & 0.756 & 0.951 & 0.942 \\
\hline Austria & 0.649 & 0.763 & 0.886 & 0.938 & & \\
\hline Bangladesh & & & 0.741 & & 0.601 & 0.615 \\
\hline Barbados & 0.746 & 0.949 & 0.866 & 0.901 & 0.796 & 0.691 \\
\hline Belgium & 0.156 & 0.167 & 0.646 & 0.768 & & \\
\hline Bolivia & & & 0.742 & 0.783 & 0.878 & 0.882 \\
\hline Botswana & & & 0.984 & & 0.965 & 0.932 \\
\hline Brazil & & & 0.625 & 0.788 & 0.836 & 0.909 \\
\hline Bulgaria & & & 0.412 & & 0.658 & 0.791 \\
\hline Canada & 0.584 & 0.830 & 0.893 & 0.805 & 0.941 & 0.945 \\
\hline Colombia & & & 0.575 & & 0.646 & 0.421 \\
\hline Costa Rica & & & & & 0.829 & 0.835 \\
\hline Croatia & & & & & 0.823 & 0.703 \\
\hline Czech Republic & & & 0.951 & & 0.818 & 0.730 \\
\hline Denmark & 0.868 & 0.616 & 0.935 & 0.936 & 0.965 & 0.981 \\
\hline El Salvador & & & 0.428 & 0.287 & 0.604 & 0.681 \\
\hline Estonia & & & 0.450 & & 0.751 & 0.741 \\
\hline Fiji & 0.977 & 0.992 & 0.972 & 0.974 & 0.985 & 0.979 \\
\hline Finland & 0.416 & 0.614 & 0.958 & 0.962 & & \\
\hline France & 0.284 & 0.167 & 0.695 & 0.723 & & \\
\hline Georgia & & & & & 0.754 & 0.864 \\
\hline Germany & & & 0.872 & 0.929 & & \\
\hline Guatemala & & & & & 0.594 & 0.584 \\
\hline Hong Kong & 0.922 & 0.983 & 0.885 & 0.905 & 0.918 & 0.890 \\
\hline Hungary & & & & & 0.337 & 0.394 \\
\hline Iceland & & & & & 0.750 & 0.806 \\
\hline Indonesia & & & 0.751 & 0.775 & 0.404 & 0.384 \\
\hline Iran & & & 0.429 & 0.310 & 0.692 & 0.765 \\
\hline Ireland & 0.743 & 0.646 & 0.979 & 0.936 & & \\
\hline Israel & 0.802 & 0.939 & 0.866 & 0.801 & 0.996 & 0.994 \\
\hline Italy & 0.239 & 0.313 & 0.647 & 0.672 & & \\
\hline Jamaica & & & & & 0.512 & 0.402 \\
\hline Japan & 0.898 & 0.903 & 0.907 & 0.868 & 0.943 & 0.940 \\
\hline Jordan & & & 0.933 & 0.930 & 0.861 & 0.900 \\
\hline
\end{tabular}

Note: The table gives the list of countries included in our sample and the ten-year average values of $C O N S$ and $C O N S \_W$ for each of them. The reported values of $C O N S$ and $C O N S \_W$ are not those used in the econometric analysis of the article and are only intended to provide an overview of central bank preferences country by country to the reader. Euro-area member states are considered until they join the European Monetary Union. 
Table A2: Table A1 (continued): Average CONS and CONS_W

\begin{tabular}{|c|c|c|c|c|c|c|}
\hline Decade & \multicolumn{2}{|c|}{ 1980's } & \multicolumn{2}{|c|}{ 1990's } & \multicolumn{2}{|c|}{ 2000's } \\
\hline Country Name & $C O N S$ & $C O N S_{-} W$ & $C O N S$ & CONS_W & $C O N S$ & CONS_W \\
\hline Kazakhstan & & & 0.124 & & 0.746 & 0.762 \\
\hline Korea, Rep. & 0.693 & 0.904 & 0.885 & 0.894 & 0.922 & 0.886 \\
\hline Kyrgyz Republic & & & & & 0.606 & 0.469 \\
\hline Latvia & & & 0.561 & 0.379 & 0.847 & 0.890 \\
\hline Lithuania & & & 0.574 & & 0.839 & 0.862 \\
\hline Malawi & 0.601 & 0.628 & 0.417 & 0.396 & 0.511 & 0.580 \\
\hline Malaysia & & & 0.989 & 0.993 & 0.955 & 0.954 \\
\hline Mauritius & & & & & 0.634 & 0.571 \\
\hline Mexico & 0.806 & 0.884 & 0.609 & 0.733 & 0.908 & 0.883 \\
\hline Moldova & & & & & 0.409 & 0.446 \\
\hline Morocco & & & 0.884 & 0.867 & 0.927 & 0.879 \\
\hline Netherlands & 0.400 & 0.472 & 0.552 & 0.544 & & \\
\hline New Zealand & & & 0.765 & 0.690 & 0.872 & 0.864 \\
\hline Nicaragua & & & & & 0.591 & 0.509 \\
\hline Nigeria & 0.634 & 0.689 & 0.245 & 0.231 & 0.094 & 0.066 \\
\hline Norway & 0.911 & 0.914 & 0.919 & 0.959 & 0.974 & 0.965 \\
\hline Peru & & & 0.474 & 0.417 & 0.973 & 0.970 \\
\hline Philippines & 0.227 & 0.236 & 0.296 & 0.184 & 0.357 & 0.326 \\
\hline Poland & & & & & 0.806 & 0.807 \\
\hline Portugal & 0.715 & 0.795 & 0.818 & 0.856 & & \\
\hline Romania & & & & & 0.210 & 0.164 \\
\hline Russian Fed. & & & 0.162 & & 0.405 & 0.403 \\
\hline Slovak Republic & & & 0.769 & & 0.463 & 0.400 \\
\hline Slovenia & & & & & 0.297 & 0.345 \\
\hline South Africa & 0.775 & 0.630 & 0.774 & 0.679 & 0.655 & 0.734 \\
\hline Spain & 0.200 & 0.212 & 0.688 & 0.780 & & \\
\hline Sweden & 0.684 & 0.573 & 0.714 & 0.701 & 0.898 & 0.939 \\
\hline Switzerland & 0.472 & 0.451 & 0.857 & 0.906 & 0.970 & 0.977 \\
\hline Thailand & & & 0.961 & & 0.834 & 0.741 \\
\hline Trinidad and Tob. & 0.934 & 0.897 & 0.859 & 0.918 & 0.780 & 0.771 \\
\hline Tunisia & & & & & 0.726 & 0.748 \\
\hline Turkey & & & 0.948 & 0.949 & 0.755 & 0.742 \\
\hline Ukraine & & & & & 0.757 & 0.808 \\
\hline United Kingdom & & & 0.504 & 0.463 & 0.829 & 0.901 \\
\hline United States & 0.585 & 0.715 & 0.774 & 0.839 & 0.857 & 0.889 \\
\hline Zambia & & & 0.026 & 0.029 & & \\
\hline
\end{tabular}

Note: The table gives the list of countries included in our sample and the ten-year average values of CONS and CONS_W for each of them. The reported values of CONS and CONS_W are not those used in the econometric analysis of the article and are only intended to provide an overview of central bank preferences country by country to the reader. Euro-area member states are considered until they join the European Monetary Union. 


\section{Appendix 3 - Details on the methodology used to compute the "cleansed" CONS and CONS_W indexes}

To compute the "cleansed" CONS and CONS_W indexes, we adopt an econometric strategy originally developed in the literature on "tax effort". Indeed, as in our case, the actual tax revenue that a country collects is not only the result of the willingness of the tax authority. It also depends on various external factors, such as the economic, social, institutional and demographic characteristics of the country. To solve this issue, the literature on "tax effort" proposes to estimate the tax capacity of a country, obtained by regressing the actual tax revenue on external factors which are independent of the the willingness of the tax authority. Then, the difference between the actual tax revenue and its predicted value (i.e. the residuals), namely the tax capacity, corresponds to the tax effort, i.e. the maximum tax revenue that a country can collect given its economic, social, institutional and demographic characteristics.

In line with this approach, we regress each of our indicators of central banks' preferences, i.e. CONS and CONS_W indexes, on a set of factors that can potentially impact the inflation and output gap volatility, regardless the conscious willingness of the central bank to prioritize inflation stabilization. Due to the censored nature of the CONS and CONS_W indexes, we consider a Tobit model, which ensures that the predicted values of the indexes are comprised in the same interval $[0,1]$. The model is estimated using a random effects estimator. In comparison to the fixed effects estimator, the main advantage of the random effects estimator is to attribute only a part of the unobserved heterogeneity to structural factors.

Formally, we estimate the following equation:

$$
C B P_{i, t}=\beta_{0}+\beta_{1} M P I_{i, t}+\beta_{2} F O_{i, t}+\beta_{3} E R S_{i, t}+\beta_{4} D G S_{i, t}+\epsilon_{i, t}
$$

where $C B P_{i, t}$ represents alternatively one of our measures of central banks' preferences, CONS and CONS_W, for country $i$ in time t. $M P I_{i, t}, F O_{i, t}$ and $E R S_{i, t}$ are variables capturing the trilemma configuration, i.e. the monetary policy independence vis-à-vis the rest of the world, the financial openness and the exchange rate stability, respectively. These variables, bounded between 0 and 1, are taken from Aizenman et al. (2013). More precisely, monetary policy independence corresponds to the correlation between interest rates of the home country and the country to which monetary policy is the most closely linked. Financial openness corresponds to the Chinn-Ito index (Chinn and Ito, 2008), and exchange rate stability is based on the standard deviation of the exchange rate of the local currency against a benchmark country's currency. $D G S_{i, t}$ represents the discretionary government spending. It is calculated following the methodology proposed by Ambrosius (2017). As it captures the changes in fiscal policy that only results from the willingness of the government, this variable aims to gauge the government's preferences. $\beta_{0}$ is the constant, and $\beta_{k}$ are the parameters associated with the independent variables. Their estimated values are reported in Table A3. Finally, $\epsilon_{i, t}$ is the error term, which theoretically corresponds to the "clean" proxy for central banks' preferences. The residuals are rescaled between 0 and 1 .

As the Figure A4 suggests, the correlation between the original indexes and their cleansed version appears very high ${ }^{28}$. Such high correlations clearly indicate that our

\footnotetext{
${ }^{28}$ The correlation between CONS and "cleansed" CONS is strongly significant and equal to $93.22 \%$, while in the case of CONS_W, the correlation is equal to $94.71 \%$.
} 
original measures of central banks' preferences are good proxies for central banks' inflation aversion.

Table A3: "Cleansed" central banks" preferences indexes : Results of the identification regression

\begin{tabular}{l|ll}
\hline \hline Dependent variable & CONS & CONS_W \\
\hline Monetary independence (MPI) & $-0.123^{* * *}$ & $-0.070^{*}$ \\
& $(0.035)$ & $(0.039)$ \\
Financial openness (FO) & $0.253^{* * *}$ & $0.242^{* * *}$ \\
& $(0.031)$ & $(0.036)$ \\
Exchange Rate Stability (FER) & -0.050 & -0.038 \\
& $(0.031)$ & $(0.035)$ \\
Discretionary Spendings (DGS) & -0.015 & -0.016 \\
& $(0.053)$ & $(0.057)$ \\
Constant & $0.615^{* * *}$ & $0.609^{* * *}$ \\
& $(0.040)$ & $(0.046)$ \\
\hline Observations & 1,127 & 981 \\
Number of countries & 77 & 71 \\
\hline \hline Note: Standard errors are reported in parentheses. & *, ${ }^{* *}$ and ${ }^{* * *}$ \\
denote significance at the $10 \%, 5 \%$ and $1 \%$ level, respectively.
\end{tabular}
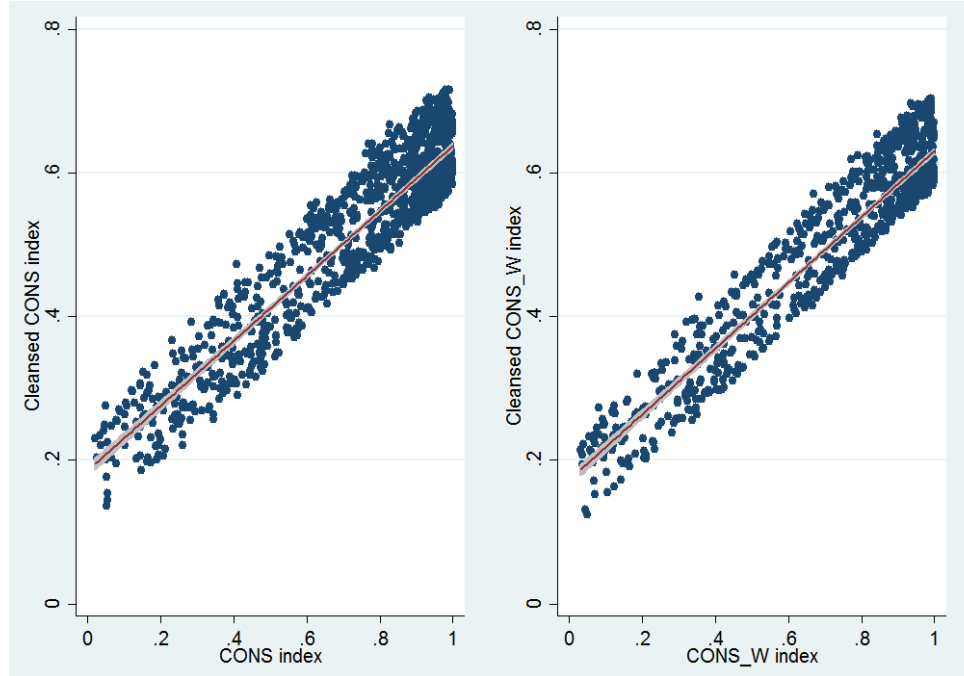

Figure A4: Correlations between initial and "cleansed" indexes of central banks' preferences. 


\section{References}

Abiad, A., Balakrishnan, R., Koeva-Brooks, P., Leigh, D., and Tytell, I. (2009). What's the damage? Medium-term output dynamics after banking crises. IMF Working Paper 09/245, International Monetary Fund.

Abiad, A., Detragiache, E., and Tressel, T. (2010). A new database of financial reforms. IMF Staff Papers, International Monetary Fund, 57(2):281-302.

Abiad, A., Leigh, D., and Mody, A. (2007). International finance and income convergence: Europe is different. IMF Working Paper 07/64, International Monetary Fund.

Aizenman, J., Chinn, M., and Ito, H. (2013). The "impossible trinity" hypothesis in an era of global imbalances: Measurement and testing. Review of International Economics, 21(3):447-458.

Aklin, M. and Kern, A. (2016). Is central bank independence always a good thing? SSRN Working Paper.

Ambrosius, C. (2017). What explains the speed of recovery from banking crises? Journal of International Money and Finance, 70:257-287.

Barth, J., Caprio, G., and Levine, R. (2004). Bank regulation and supervision: What works best? Journal of Financial Intermediation, 13(2):205-248.

Bayoumi, T., Dell'Ariccia, G., Habermeier, K., Mancini-Griffoli, T., and Valencia, F. (2014). Monetary policy in the new normal. IMF Staff Discussion Note 14/3, International Monetary Fund.

Beck, T., Demirgüç-Kunt, A., and Levine, R. (2010). Financial institutions and markets across countries and over time: The updated financial development and structure database. World Bank Economic Review, 24(1):77-92.

Berger, W. and Kißmer, F. (2013). Central bank independence and financial stability: A tale of perfect harmony? European Journal of Political Economy, 31:109-118.

Bernanke, B. (2013). A century of US central banking: Goals, frameworks, accountability. Journal of Economic Perspectives, 27(4):3-16.

Bernanke, B. and Gertler, M. (2000). Monetary policy and asset price volatility. NBER Working Paper No. 7559, National Bureau of Economic Research.

BIS (2009). Issues in the governance of central banks. A Report from the Central Bank Governance Group of the BIS.

BIS (2014). Basel III leverage ratio framework and disclosure requirements. A Report from the Basel Committee on Banking Supervision.

Blanchard, O. and Galí, J. (2007). Real wage rigidities and the new Keynesian model. Journal of Money, Credit and Banking, 39(1):35-65.

Blanchard, O. and Quah, D. (1989). The dynamic effects of aggregate demand and supply disturbances. American Economic Review, 79(4):655-673. 
Blot, C., Creel, J., Hubert, P., Labondance, F., and Saraceno, F. (2015). Assessing the link between price and financial stability. Journal of Financial Stability, 16:71-88.

Bordo, M., Eichengreen, B., Klingebiel, D., Martinez-Peria, M., and Rose, A. (2001). Is the crisis problem growing more severe? Economic Policy, 16(32):53-82.

Bordo, M. and Wheelock, D. (1998). Price stability and financial stability: The historical record. The Federal Reserve Bank of Saint Louis Review, (September/October):41-62.

Borio, C. (2014a). The financial cycle and macroeconomics: What have we learnt? Journal of Banking \& Finance, 45:182-198.

Borio, C. (2014b). Monetary policy and financial stability: What role in prevention and recovery? BIS Working Paper No. 440, Bank for International Settlements.

Borio, C. and Lowe, P. (2004). Securing sustainable price stability: Should credit come back from the wilderness? BIS Working Paper No. 15\%, Bank for International Settlements.

Borio, C. and Zhu, H. (2012). Capital regulation, risk-taking and monetary policy: A missing link in the transmission mechanism? Journal of Financial Stability, $8(4): 236-251$.

Brainard, W. (1967). Uncertainty and the effectiveness of policy. American Economic Review, 57(2):411-425.

Calvo, G., Izquierdo, A., and Mejía, L. (2008). Systemic sudden stops: The relevance of balance-sheet effects and financial integration. NBER Working Paper No. 14026, National Bureau of Economic Research.

Caprio, G., D'Apice, V., Ferri, G., and Puopolo, G. (2014). Macro-financial determinants of the great financial crisis: Implications for financial regulation. Journal of Banking \& Finance, 44:114-129.

Chinn, M. and Ito, H. (2008). A new measure of financial openness. Journal of Comparative Policy Analysis, 10(3):309-322.

Chortareas, G., Logothetis, V., Magkonis, G., and Zekente, K. (2016). The effect of banking supervision on central bank preferences: Evidence from panel data. Economics Letters, 140:11-13.

Christiano, L., Ilut, C., Motto, R., and Rostagno, M. (2010). Monetary policy and stock market booms. NBER Working Paper No. 16402, National Bureau of Economic Research.

Čihák, M. (2007). Central bank independence and financial stability. International Monetary Fund, Mimeo.

Čihák, M. and Schaeck, K. (2010). How well do aggregate prudential ratios identify banking system problems? Journal of Financial Stability, 6(3):130-144.

Claessens, S. and Laeven, L. (2004). What drives bank competition? Some international evidence. Journal of Money, Credit and Banking, 36(3):563-583. 
Claessens, S. and Van Horen, N. (2014). Foreign banks: Trends and impact. Journal of Money, Credit and Banking, 46(1):295-326.

Cukierman, A. (2011). Reflections on the crisis and on its lessons for regulatory reform and for central bank policies. Journal of Financial Stability, 7(1):26-37.

Cukierman, A., Web, S., and Neyapti, B. (1992). Measuring the independence of central banks and its effect on policy outcomes. World Bank Economic Review, 6(3):353-398.

De Paoli, B. and Paustian, M. (2017). Coordinating monetary and macroprudential policies. Journal of Money, Credit and Banking, 49(2-3):319-349.

Dell'Ariccia, G., Igan, D., Laeven, L., and Tong, H. (2014). Policies for macrofinancial stability: Dealing with credit booms and busts. Financial Crises: Causes, Consequences, and Policy Responses, pages 325-364.

Demirgüç-Kunt, A. and Detragiache, E. (1998). The determinants of banking crises in developing and developed countries. IMF Staff Papers, International Monetary Fund, 45(1):81-109.

Demirgüç-Kunt, A., Detragiache, E., and Tressel, T. (2008). Banking on the principles: Compliance with basel core principles and bank soundness. Journal of Financial Intermediation, 17(4):511-542.

DeYoung, R. and Roland, K. (2001). Product mix and earnings volatility at commercial banks: Evidence from a degree of total leverage model. Journal of Financial Intermediation, 10(1):54-84.

Di Noia, C. and Di Giorgio, G. (1999). Should banking supervision and monetary policy tasks be given to different agencies? International Finance, 2(3):361-378.

Domaç, I. and Martinez Peria, M. (2003). Banking crises and exchange rate regimes: Is there a link? Journal of International Economics, 61(1):41-72.

Doumpos, M., Gaganis, C., and Pasiouras, F. (2015). Central bank independence, financial supervision structure and bank soundness: An empirical analysis around the crisis. Journal of Banking \& Finance, 61:S69-S83.

Dreher, A., Sturm, J., and de Haan, J. (2008). Does high inflation cause central bankers to lose their job? Evidence based on a new data set. European Journal of Political Economy, 24(4):778-787.

Fazio, D., Tabak, B., and Cajueiro, D. (2015). Inflation targeting: Is IT to blame for banking system instability? Journal of Banking 83 Finance, 59:76-97.

Franck, R. and Krausz, M. (2008). Why separate monetary policy from banking supervision? Journal of Comparative Economics, 36(3):388-411.

Frankel, J. and Saravelos, G. (2012). Can leading indicators assess country vulnerability? Evidence from the 2008-09 global financial crisis. Journal of International Economics, 87(2):216-231. 
Frankel, J., Schmukler, S., and Servén, L. (2004). Global transmission of interest rates: Monetary independence and currency regime. Journal of International Money and Finance, 23(5):701-733.

Frappa, S. and Mésonnier, J. (2010). The housing price boom of the late 1990s: Did inflation targeting matter? Journal of Financial Stability, 6(4):243-254.

Gadanecz, B., Miyajima, K., and Urban, J. (2015). Introducing financial stability considerations into Taylor rules in emerging market economies. Applied Economics Letters, 22(16):1320-1324.

Garriga, A. (2016). Central bank independence in the world: A new data set. International Interactions, 42(5):849-868.

Ghosh, A., Ostry, J., and Tsangarides, C. (2011). Exchange rate regimes and the stability of the international monetary system. Occasional Paper No. 270, International Monetary Fund.

Giannone, D., Lenza, M., and Reichlin, L. (2011). Market freedom and the global recession. IMF Economic Review, 59(1):111-135.

Giese, J., Andersen, H., Bush, O., Castro, C., Farag, M., and Kapadia, S. (2014). The credit-to-GDP gap and complementary indicators for macroprudential policy: Evidence from the UK. International Journal of Finance $\&$ Economics, 19(1):25-47.

Goodhart, C. and Schoenmaker, D. (1995). Institutional separation between supervisory and monetary agencies. In The Central Bank and the Financial System, pages 333-413. Palgrave Macmillan, London.

Hasan, I. and Mester, L. (2008). Central bank institutional structure and effective central banking: Cross-country empirical evidence. Comparative Economic Studies, 50(4):620-645.

Heller, R. (1991). Prudential supervision and monetary policy. In Frenkel, J. and Goldstein, M., editors, Essays in Honor of Jacques Polak, pages 269-281. International Monetary Fund, Washington DC.

Ioannidou, V. (2005). Does monetary policy affect the central bank's role in bank supervision? Journal of Financial Intermediation, 14(1):58-85.

Issing, O. (2003). Monetary and financial stability: Is there a trade-off? Conference on "Monetary Stability, Financial Stability and theBusiness Cycle", March 28-29, 2003, Bank for International Settlements, Basel.

Jiménez, G., Ongena, S., Peydró, J., and Saurina, J. (2014). Hazardous times for monetary policy: What do twenty-three million bank loans say about the effects of monetary policy on credit risk-taking? Econometrica, 82(2):463-505.

Kaminsky, G. and Schmukler, S. (2003). Short-run pain, long-run gain: The effects of financial liberalization. NBER Working Paper No. 9787, National Bureau of Economic Research.

Khan, A. (2017). Central bank legal frameworks in the aftermath of the global financial crisis. IMF Working Paper 17/101, International Monetary Fund. 
Kindleberger, C. (1978). Manias, Panics, and Crashes: A History of Financial Crises. Palgrave Macmillan.

King, M. (1997). Changes in UK monetary policy: Rules and discretion in practice. Journal of Monetary Economics, 39(1):81-97.

Klomp, J. and de Haan, J. (2009). Central bank independence and financial instability. Journal of Financial Stability, 5(4):321-338.

Koetter, M., Roszbach, K., and Spagnolo, G. (2014). Financial stability and central bank governance. International Journal of Central Banking, 10(4):31-67.

Köhler, M. (2015). Which banks are more risky? The impact of business models on bank stability. Journal of Financial Stability, 16:195-212.

La Porta, R., Lopez-De-Silanes, F., and Shleifer, A. (2002). Government ownership of banks. Journal of Finance, 57(1):265-301.

Laeven, L. and Levine, R. (2009). Bank governance, regulation and risk taking. Journal of Financial Economics, 93(2):259-275.

Laeven, L. and Valencia, F. (2013). Systemic banking crises database. IMF Economic Review, 61(2):225-270.

Lambertini, L., Mendicino, C., and Punzi, M. (2013). Leaning against boom-bust cycles in credit and housing prices. Journal of Economic Dynamics and Control, 37(8):1500-1522.

Lane, P. and Milesi-Ferretti, G. (2007). The external wealth of nations mark II: Revised and extended estimates of foreign assets and liabilities, 1970-2004. Journal of International Economics, 73(2):223-250.

Laséen, S., Pescatori, A., and Turunen, J. (2017). Systemic risk: A new trade-off for monetary policy? Journal of Financial Stability, 32:70-85.

Levieuge, G. and Lucotte, Y. (2013). Les cibleurs d'inflation sont-ils monomaniaques ? Revue Française d'Économie, 28(4):49-81.

Levieuge, G. and Lucotte, Y. (2014). A simple empirical measure of central banks' conservatism. Southern Economic Journal, 81(2):409-434.

Lin, S. (2010). On the international effects of inflation targeting. The Review of Economics and Statistics, 92(1):195-199.

Lombardi, D. and Siklos, P. (2016). Benchmarking macroprudential policies: An initial assessment. Journal of Financial Stability, 27:35-49.

Lucas, R. (2000). Inflation and welfare. Econometrica, 68(2):247-274.

Minsky, H. (1972). Financial stability revisited: The economics of disaster. In Board of Governors of the Federal Reserve System, editor, Reappraisal of the Federal Reserve Discount Mechanism, volume 3, pages 95-136. Washington, D.C: Federal Reserve Board. 
Mishkin, F. (2017). Rethinking monetary policy after the crisis. Journal of International Money and Finance, 73(Part B):252-274.

Mundell, R. (1963). Capital mobility and stabilization policy under fixed and flexible exchange rates. The Canadian Journal of Economics and Political Science, $29(4): 475-485$.

Oosterloo, S. and de Haan, J. (2004). Central banks and financial stability: A survey. Journal of Financial Stability, 1(2):257-273.

Petreski, M. (2014). Inflation targeting at the crossroads: Evidence from postcommunist economies during the crisis. Communist and Post-Communist Studies, $47(2): 247-260$.

Ratnovski, L. and Huang, R. (2009). Why are Canadian banks more resilient? IMF Working Paper 09/152, International Monetary Fund.

Reis, R. (2013). Central bank design. Journal of Economic Perspectives, 27(4):17-44.

Rogoff, K. (1985). The optimal degree of commitment to an intermediate monetary target. Quarterly Journal of Economics, 100(4):1169-1189.

Schaeck, K. and Čihák, M. (2014). Competition, efficiency, and stability in banking. Financial Management, 43(1):215-241.

Schularick, M. and Taylor, A. (2012). Credit booms gone bust: Monetary policy, leverage cycles, and financial crises, 1870-2008. American Economic Review, 102(2):10291061.

Schwartz, A. (1995). Why financial stability depends on price stability. Economic Affairs, 15(4):21-25.

Shimpalee, P. and Breuer, J. (2006). Currency crises and institutions. Journal of International Money and Finance, 25(1):125-145.

Siklos, P. (2002). The changing face of central banking: Evolutionary trends since World War II. Cambridge University Press.

Smaga, P. (2013). Assessing involvement of central banks in financial stability. Center for Financial Stability Policy Paper.

Smets, F. (2014). Financial stability and monetary policy: How closely interlinked? International Journal of Central Banking, 10(2):263-300.

Stremmel, H. and Zsámboki, B. (2015). The relationship between structural and cyclical features of the EU financial sector. ECB Working Paper No. 1812, European Central Bank.

Svensson, L. (2012). Comment on Michael Woodford "Inflation targeting and financial stability". Sveriges Riksbank Economic Review, 1:33-39.

Taylor, J. (1979). Estimation and control of a macroeconomic model with rational expectations. Econometrica, 47(5):1267-1286. 
Toniolo, G. and White, E. (2015). The evolution of the financial stability mandate: From its origins to the present day. NBER Working Paper No. 20844, National Bureau of Economic Research.

Ueda, K. and Valencia, F. (2014). Central bank independence and macro-prudential regulation. Economics Letters, 125(2):327-330.

Van Leuvensteijn, M., Bikker, J., Van Rixtel, A., and Kok Sørensen, C. (2011). A new approach to measuring competition in the loan markets of the Euro area. Applied Economics, 43(23):3155-3167.

Whelan, K. (2013). A broader mandate: Why inflation targeting is inadequate. In Reichlin, L. and Baldwin, R., editors, Is inflation targeting dead? Central Banking After the Crisis, pages 104-112. CEPR, VoxEU.org Book.

White, W. (2006). Is price stability enough? BIS Working Paper No. 205, Bank for International Settlements.

Woodford, M. (2003). Interest and Prices: Foundations of a Theory of Monetary Policy. Princeton University Press.

Woodford, M. (2012). Inflation targeting and financial stability. NBER Working Paper No. 17967, National Bureau of Economic Research. 